The Journal of Architecture 16, no. 2 (2011): 231-265

\title{
MUTUAL VISIBILITY OF POINTS IN BUILDING PLANS
}

\author{
Mahbub Rashid, PhD, RA \\ Associate Professor of Architecture \\ School of Architecture, Design, and Planning, University of Kansas \\ 1465 Jayhawk Boulevard \\ Lawrence, Kansas 66045 \\ E-mail: mrashid@ku.edu
}




\title{
MUTUAL VISIBILITY OF POINTS IN BUILDING PLANS
}

\begin{abstract}
In this paper, attempts are made to characterize building plans based on mutual visibility of points defined by the lines composing these plans. Since lines in building plans represent real walls and their surfaces, points defined by these lines are considered to have certain perceivable significance. The paper considers two overlapping sets of points-vertices and coordination points. Vertices are tangible points defined by the free ends and the intersections of lines, while coordination points are the tangible and intangible points defined by intersecting lines and/or diagonals. A coordination point helps coordinate two or more points, either physically by direct access or visually by sightlines. The paper proposes graphtheoretic techniques to characterize vertices and coordination points based on their visual relations defined in a number of ways. These techniques are applied to three sets of artificial building plans representing the cellular, deformed, and free plan types-types that are quite common in architectural literature. With the help of these techniques, several elusive properties of architectural space and experience are formally described highlighting similarities and differences among these plan types. The significance of these techniques is discussed in relation to architecture, perceptual psychology, and cognitive science.
\end{abstract}

\section{INTRODUCTION}

When inside a building, one rarely gets to see the building all at once or as a whole. Therefore, one is required to construct any global understanding of the building based on its local features. Gibson's theory of ecological visual perception outlines how this process of bottom-up understanding might work. ${ }^{1,2}$ According to Gibson, our perception of the environment depends not just on any kind of stimulus related to sensations. Rather, it depends only on the "meaningful stimulus" which "corresponds to the permanent properties of the environment." ${ }^{3}$ Gibson uses the term "invariants" to refer to these permanent properties of the environment. According to Gibson, in the first stage of the study of perception, perceptual psychologists must find ways to describe the structures of invariants that specify the environment. In the second stage, they must study the empirical relevance of these structures; and once the empirically valid structures are identified, in the third stage, they must study how human beings accomplish the task of constructing these structures.

It is Interesting to note that geometry, psychology/cognition, and the ways we perform spatial reasoning using natural language already suggest a common way to define these perceptual invariants. In trying to 
find out which geometry fits the perceptual needs of an observer inside buildings, it is interesting to go back to the history and philosophy of geometry. In 1872, in his lecture at the University of Erlangen, now known as the Erlanger Program, Felix Klein proposed a unifying principle for classifying various geometries based on automorphisms, i.e., one-to-one transformations of objects in the space onto itself preserving the basic relations among the objects. ${ }^{4}$ As a result, he created a hierarchy of geometries with topology at the root. In his program, all other geometries can be obtained by adding axioms to topology and, therefore, one class of geometry can be seen as a more specialized case of another class. Properties that are preserved in a certain group of transformations are called invariants. In general, a certain group of transformations is strictly contained in the preceding group of transformations: for example, all affine transformations are also projective transformations and therefore preserve projective invariants. An understanding of invariants of each class of geometry in the hierarchy, thus, seems to be important for describing a building from the viewpoint of an observer located inside the building.

Like the geometry, human beings may follow a similar process in the construction of cognitive space (i.e., the representational space) from perception and experience. Jean Piaget, with his colleague Berbel Inhelder, conducted the groundwork on the topic. ${ }^{5,6,7}$ In Piaget's opinion, the representational space is different from the perceptual space. The perceptual space may be assumed the same for all human beings, but the representational space is different for children at different stages of the development of the intelligence. To be able to represent space, the child must learn to coordinate spatial relationships mentally through a process that proceeds from the more elementary to the more complicated aspects of space. In this regard, Piaget's experiments show that fundamental spatial notions are not the basic elements of the Euclidean geometry (such as lines and angles), but topological concepts (such as connectedness, inclusion and order). The conclusion of Piaget's work is that the representational space of the child starts with elementary topological intuitions before becoming at the same time projective and Euclidean. A psychological process transforms the topological notion of order to the projective straight line after the discovering of points of view, and to a Euclidean system when the child becomes able to understand distances and movements.

Based on the Erlanger Program and Piaget's ideas, it is possible to imagine a framework of configurational studies of buildings based on topological, projective, and metric properties. Through topology, information about neighborliness, connections, and the presence/absence of holes of the object will be provided for these studies. Through projective geometry, information about convexity/concavity of the object will be provided. Finally, through metrics or Euclidean geometry, information about compactness, symmetry, and so on will be provided. As essential problem here is how to parse the topological, projective and metric properties in the configurational studies of buildings from the viewpoint of an observer located inside them. This problem can probably be solved by the way we perform 
everyday spatial reasoning using natural language. It would seem that in everyday spatial reasoning, we depend more on topology and projective geometry and less on Euclidean geometry. That is because as observers we are not very good at using exact metric and global properties to describe the environment, whereas we can very easily perform context-dependent comparisons or understand relational properties. Naturally then, as observers we use terms such as "directly connected to", "adjacent to", "in-between," etc., referring to the topological, and "in front of", "out of sight", "within sight," etc., referring to the projective relations between various aspects of the object as well as between various aspects of the object and us.

Consequently, both architecture and cognitive science have frequently used projective elements/relations in conjunction with topology to explain how observers may perceive and understand buildings while on the ground. ${ }^{8}$ In architecture, space syntax theories and techniques have been most successful in this regard. Space syntax uses the convex unit and the axial line as the basic units of description, because they are the elementary projective units immediately available to the observer. (For earlier definitions of these units, see Hillier and Hanson. ${ }^{9}$ For more recent definitions, see Peponis et al. ${ }^{10,11,12}$ ) Space syntax also uses the 360 -degree visual polygon available from a point, called an isovist, ${ }^{13}$ as a unit of description. e.g.,14,15,16,17 The two most important techniques developed based on convex and axial units that space syntax has used to describe the global structure of an observer's visual experience of buildings are the convex and axial maps. Earlier, the convex map was defined as the fewest number of fattest convex spaces and the axial map as the fewest number of longest axial lines that cover a spatial system. ${ }^{18}$ Once building plans are expressed as convex or axial maps, space syntax uses graph-theoretic techniques to describe the topological relationships among the spatial units in these maps. More recently, a well-defined set of visual polygons and their topological relationships, known as the visibility graph analysis, have also been used by space syntax to describe an observer's visual experience of buildings. ${ }^{19}$ Taken together, the topological properties of the convex map, the axial map, and visual polygons defined using graph-theoretic techniques are able to provide a rich description of how observers perceive and experience buildings.

Like architecture, in cognitive science place and view graphs have been used as models of mental representations of environments. In the place graph, nodes correspond to single places or positions within an environment, edges describe the connectivity between nodes. In their most basic form place graphs are topological representations of space, in which nodes carry local position information, and edges carry local navigation rules, such as 'turn left' or 'follow road', that allow navigating between nodes. In the view graph, each node corresponds to a pictorial snapshot of the environment as seen while walking through the environment. Nodes are connected by edges if the corresponding views can occur in 
immediate sequence while walking. They are labelled with local navigation rules. The basic idea of the view graph is to generalize route memories to a more flexible representation of space. ${ }^{20}$

Whether it is the convex map, the axial map, and the visibility graph analysis of space syntax, or the view and place graphs of spatial cognition, these techniques appear particularly promising for explaining the process of perception and understanding of buildings by observers on the ground. They allow an efficient representation of the environment at a wide range of scale, and at the same time they are capable and flexible enough to retain a substantial amount of psychologically and behaviourally relevant information of the environment. It is for this reason this paper uses some of the graph-theoretic techniques of space syntax on the elements defining the boundary of the environment to describe yet another layer of global properties of the environment that may be psychologically and behaviourally relevant. However, unlike the convex map, the axial map, and the isovists, whose relevance to spatial cognition was hardly backed initially by the empirical findings and models of spatial representation in animal and human brains, the importance of environmental boundaries in spatial cognition seems to be well founded in neuroscience. $^{21,22}$

Much of the evidence concerning the importance of environmental boundaries in spatial cognition comes from the studies on "place cells". Studies suggest that the firing of these cells in the hippocampus of rats ${ }^{23}$ and primates ${ }^{24}$ is able to provide a neural representation of the animal's location relative to the surrounding environment. O'keefe and Burgess ${ }^{25}$ observed that the location of peak firing of a given place cell typically remained in a constant position relative to the nearest boundary, and several of the firing fields were stretched along the axes of the environment defined by its boundaries. In contrast to the robust effect of environmental boundaries on place cell firing, discrete landmarks within an environment have very little effect on place cell firing. ${ }^{26}$ Further, removing environmental boundaries tended to lead to destruction of the place cell response, while removing individual distal cues to orientation did not have

any significant effects on the response. ${ }^{27,28}$ In light of the importance of environmental boundaries in spatial cognition, this paper will focus on the patterns of mutual visibility of some of the elements defining environmental boundaries.

\section{EXPLAINING THE LOCAL UNDERSTANDING OF THE SHAPE AND CONFIGURATION OF BUILDINGS BASED ON VISIBILITY OF SURFACES AND THEIR EDGES}

Buildings are composed of boundaries or walls with one or more real surfaces that are humanly perceivable. These boundaries have edges either at their free ends or at places where they intersect other walls. Building plans represent these walls as lines and their edges as vertices or endpoints. As a 
matter of convention, we retrieve many perceivable qualities of buildings from their plans. The purpose of this paper is to study the relations of mutual visibility among a set of points defined by the geometry of the lines of a building plan. Mutual visibility of points in a building plan is interesting for it may help illustrate how one, as an observer on the ground, reads a building based on how one identifies its surfaces and edges and their visible relations.

How the patterns of visibility of surfaces and their edges affect our perception and understanding of spatial units in buildings can be made clear with the help of an isovist-a 360-degree visual field available from a position-in a set of simple building plans shown in figures 1-3. These plans show three generic ways in which lines representing walls with real surfaces may help define spaces in buildings. In the first set of plans, the spatial units are defined by lines representing walls as fixed-partitions. In the second set of plans, the spatial units are defined by lines representing freestanding partitions. In the third set, the spatial units are defined by lines representing a deformed boundary. The reasons why these three generic plan types are used in this study will be discussed later.

Figures 4-6 show each of the plans in figures 1-3 with an isovist drawn from one position in one of the two spaces. Call the space containing the position the 'origin space'. The isovist boundary in figure 4.1 includes every line defining the origin space completely. The isovist also does not leak out at any of the corners of the origin space. As a result, the origin space appears to be well-contained. The isovist spans out to the adjacent space without affecting the unitary quality of the origin space because the boundary of the isovist in the adjacent space does not include a corner or a vertex, hence appears to extend infinitely like the horizon. In figure 4.2, again, the origin space appears to be well-contained because the isovist does not leak out at any of the corners of the space. However, the isovist spanning out to the adjacent space now seems to affect the unitary quality of the origin space, because the boundary of the isovist includes a corner or a vertex defined by two partly visible lines implying at least two surfaces of the adjacent space. In figure 4.3, a significant part of the isovist now spans out to the adjacent space, and its boundary picks up two corners and three lines representing in full or in part three surfaces of the adjacent space. As a result, the unitary quality of both the spaces is destroyed, and these spaces begin to read as one large space instead of two smaller spaces.

Now consider the origin space in figure 5.1. Even though the boundary of the isovist includes every line defining the origin space completely, the space does not appear to be as well-contained as that in figure 4.1 or figure 4.2. That is because the isovist leaks out at least at two of the four defining corners of the origin space suggesting a sense of flow along the lines at these corners-a phenomenon often associated with instances where ones sees a surface of a building but not its edges. Any mystery of freeflow at the undefined corners of the origin space however is somewhat lost in figure 5.2, because now one of the two leaked-out segments of the isovist picks up a corner of the adjacent space. As a result, the 
origin space may now feel as if it is a part of a bigger whole. In figure 5.3, the mystery of free-flow is lost completely. The freestanding partition reads more like a small object in a large space.

The origin space in figure 6.1 is confined, yet less so than those in figures 4.1 and $\mathbf{4 . 2}$, because its open corner allows the space to leak out. However, the space does not suggest any sense of flow along any of the two lines at the open corner, as was observed in figure 5.1, because the endpoints of these lines are visible. As was observed in figure 4.1, a part of the isovist spans out to the adjacent space without affecting the unitary quality of the origin space because the isovist boundary in the adjacent space does not include a corner or a vertex, hence appears to extend infinitely like the horizon. Compared to the origin space in figure 6.1, the origin space in figure 6.2 is less well-defined. It suggests becoming a part of a larger whole because the isovist boundary includes a corner or a vertex defined by two partly visible lines that imply at least two surfaces of the adjacent space. Finally, the origin space in figure 6.3 seemed to have lost its identity completely to a bigger whole where all but one corners remain out of the visual field.

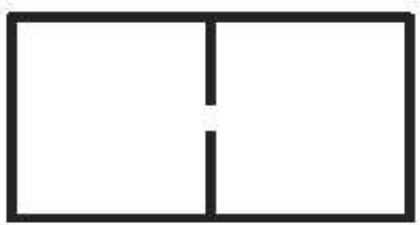

1.1

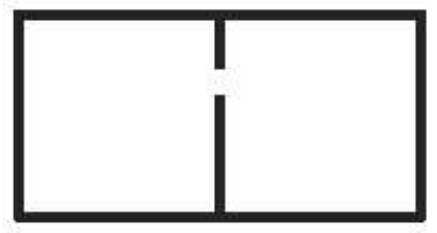

1.2

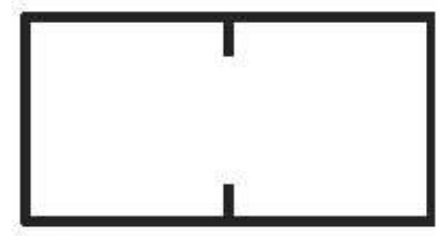

1.3

Figure 1: A set of simple building plans with fixed partitions.

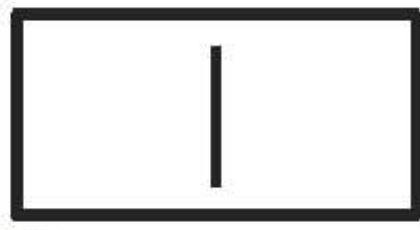

2.1

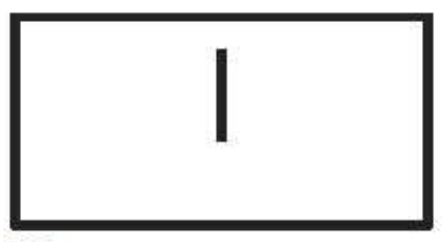

2.2

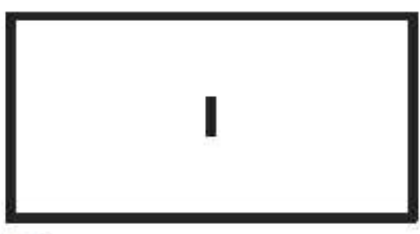

2.3

Figure 2: A set of simple building plans with free-standing partitions.
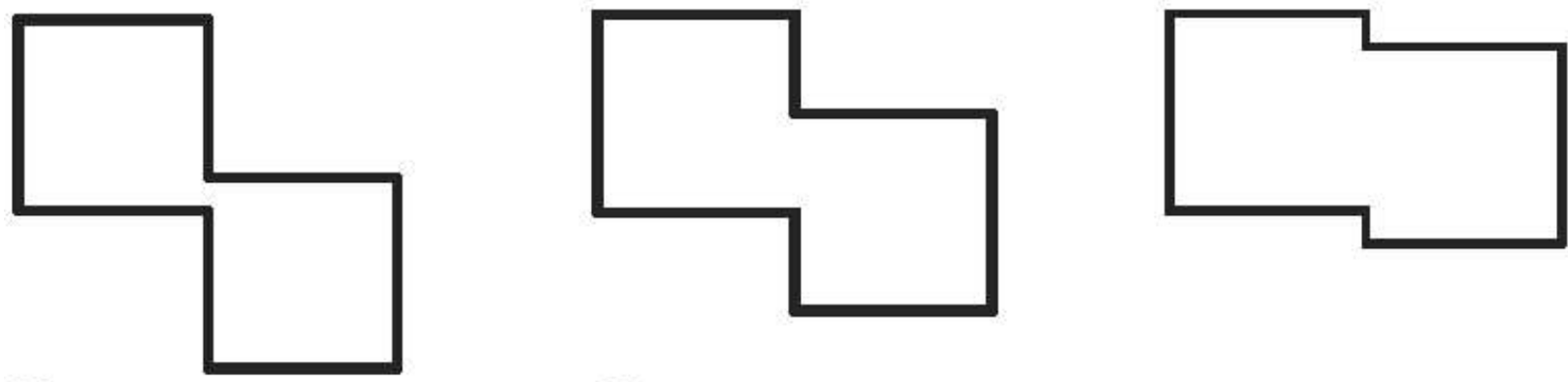

3.1

3.2

3.3

Figure 3: A set of simple building plans with no partitions. Here, spaces are defined by deforming the boundary. 


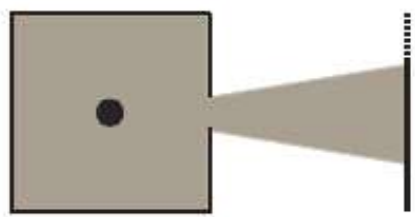

4.1

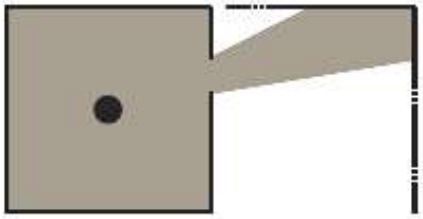

4.2

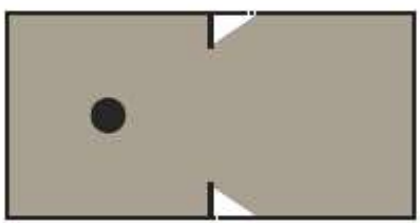

4.3

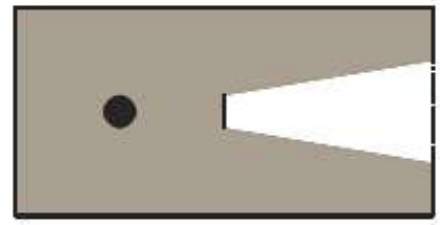

5.3

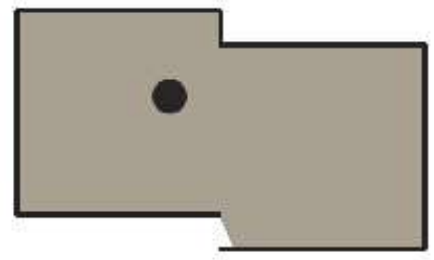

6.3

6.1

6.2

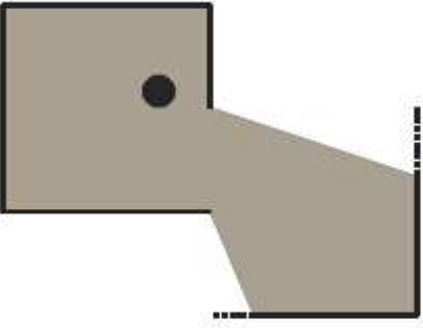

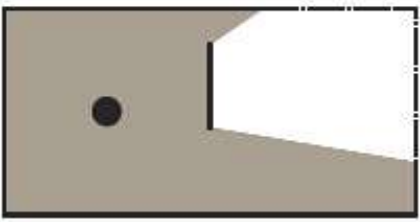

5.2

Figure 5: An isovist drawn from the same position in each plan.

Figure 6: An isovist drawn from the same position in each plan.

As this comparative analysis shows, visibility of the edges of real surfaces as represented by corners and endpoints of lines in a plan may affect our perception and understanding of spatial units in buildings in many ways. For example, when the boundary of an isovist contains only some part of a line but not its endpoints, the surface represented by the line suggests continuity with no immediate end just like the horizon. When the boundary of an isovist contains a corner defined by lines whose other ends remain out of sight, the surfaces represented by these lines suggest continuity as well as containment. When the boundary of an isovist in a space contains two or more corners of the space, the space appears contained.

Buildings, however, are rarely as simple as the ones shown in figures 1-3. Therefore, an observer on the ground often needs multiple isovists to see and understand a building. Since an observer is able to occupy an infinite number of positions in a space, some objective methods to describe visibility relations among endpoints or vertices in a plan would certainly be helpful given the importance of environmental boundaries in spatial cognition (see above). To be sure, these methods would be helpful for describing differences among building plans in terms of visibility relations among surface edges as represented by vertices or endpoints. They would also be helpful for identifying vertices that occupy the most integrated 
positions in a plan in terms of their visual relations. If it is assumed for a moment that visibility relations among vertices representing surface edges provides one logical way to reconstruct how an observer located inside the plan perceives and understands building plans, then it is likely that the observer would use the most integrated set of vertices as anchors for her understanding. This issue will be discussed further later in the paper.

Therefore, various techniques to describe mutual visibility of endpoints of the lines defining a building plan are discussed in this paper. It must however be noted here that all the techniques proposed in the paper are computationally demanding. No software program is available at this time to run the routines of these techniques. Therefore, the paper applies these techniques manually to analyse mutual visibility of endpoints in building plans. To keep things computationally manageable, simple artificial building plans that have some theoretical and historical significance in architecture are used in this study. Though simple in nature, these plans are not as simple as the ones shown in figures 1-3. Before presenting the techniques and the analyses of building plans using these techniques, the sample of plans included in this study will be introduced.

\section{SAMPLING BUILDING PLANS}

Regarding building plans, certain typological distinctions are frequently made in architectural literature. For example, the plans of the villas by Palladio and Ledoux are often considered cellular plans. In contrast, the plans of the houses by Le Corbusier and Mies van der Rohe are often considered free plans. These labels make intuitive sense, but they are not precise. The plans of the Villa Savoie by Le Corbusier (Figure 7) and the Farnsworth House by Mies van der Rohe (Figure 8) are very different, yet they are both considered free plans (see below). In fact, it would seem more reasonable to put the plan of the Villa Savoie somewhere between the plans of Palladio's Villas and Mies' Houses. However, the problem can be more subtle: Should the plan of Palladio's Villa in figure 9 be considered a cellular plan or a free plan? The ambiguity arises for the cross-shaped central space covers a large area of this plan.

Despite ambiguities, architectural theorists frequently make intuitive decisions about the type of a plan. In 1941, Giedion, in his Space, Time, and Architecture, characterizes F. L. Wright's house plans in the following manner:

Before I had seen any of Wright's houses, I stopped once for a rest in a hunting lodge in the Vermont hills. . The interior space [of the lodge] was undivided except for a partition which cut off the kitchen and the sleeping room. . At that moment I began to understand the way Wright conceived his interior spaces. He worked fundamentally and as far as possible with the house as 
one room. Its inner space is differentiated to meet special needs. As he pointed out, he "declared the whole ... floor as one room ... screening various partitions in the big room, for certain domestic purposes ..." 29

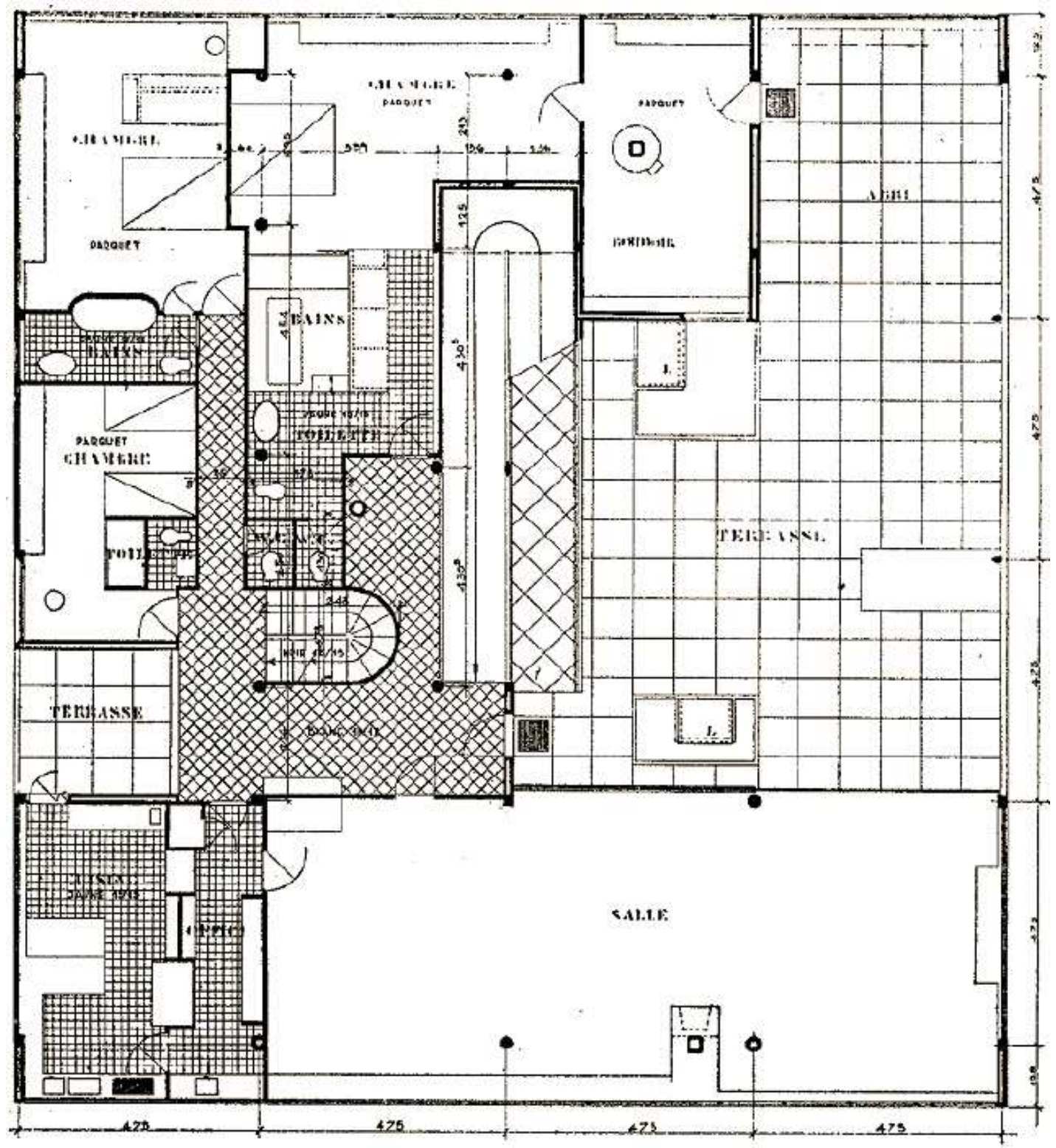

Figure 7: Plan of the main floor of Villa Savoie by Le Corbusier. Source: W Boesiger, Le Corbusier, 


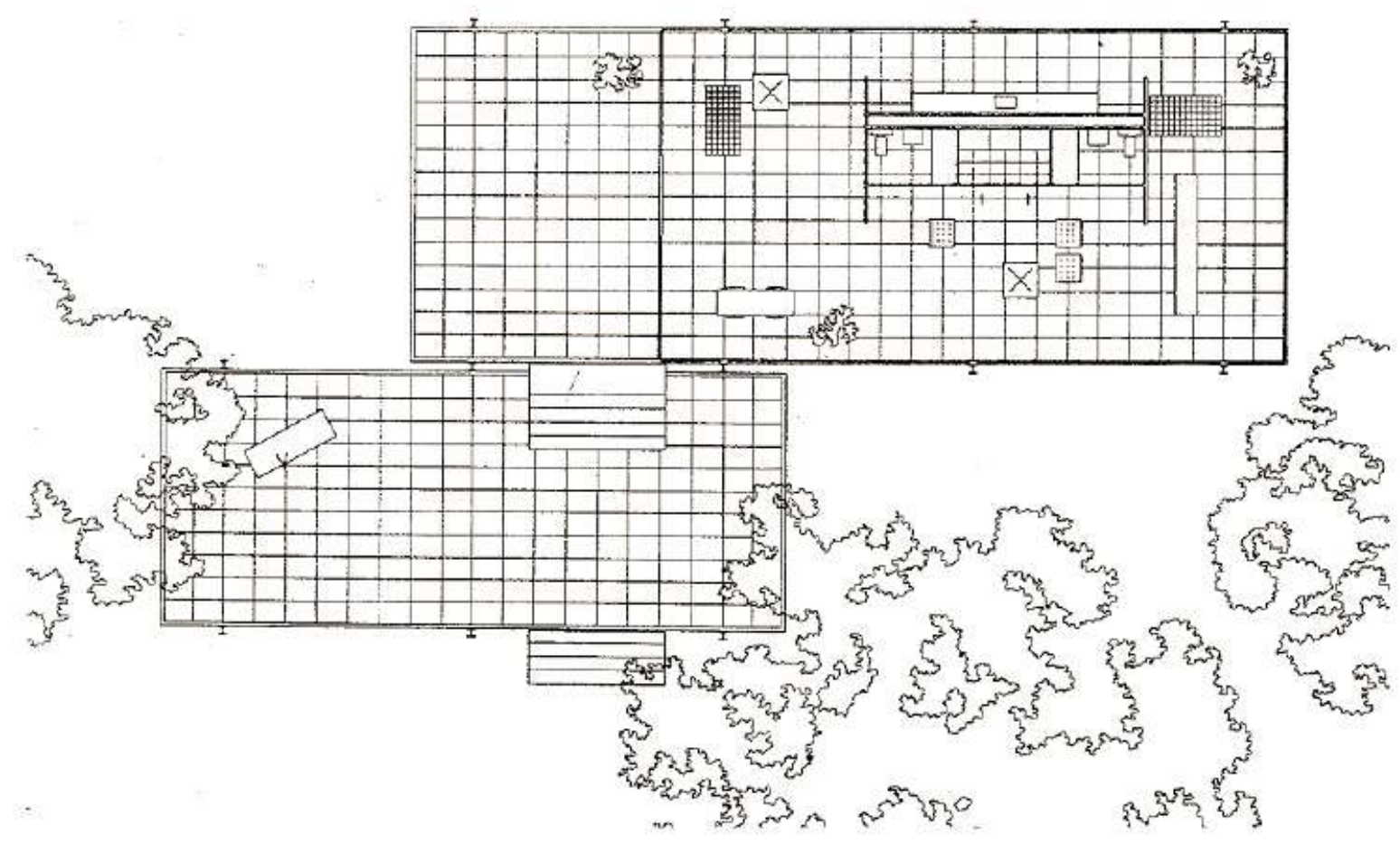

Figure 8: Plan of Farnsworth House by Mies van der Rohe. Source: D Spaeth, Mies van der Rohe (New York, Rizzoli, 1985)

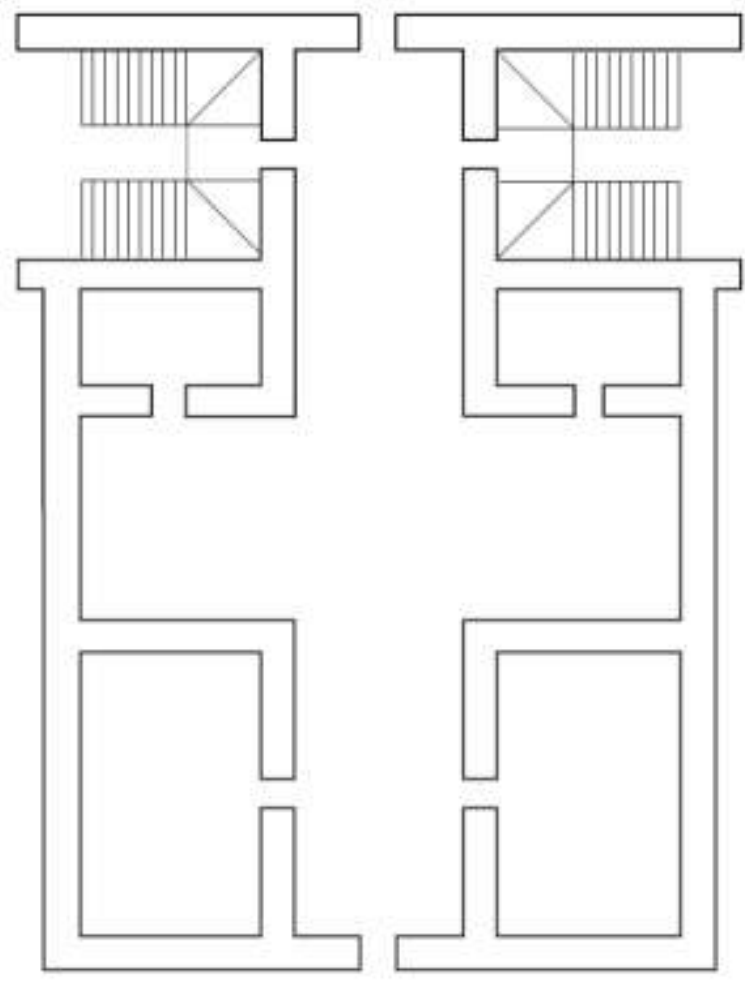

Figure 9: Plan of a villa by Palladio. Source: Palladio, The Four Books 


\section{Works and Projects (Barcelona, Editorial Gustavo Gili, S. A., 1994)}

In contrast, about Le Corbusier's Villa Savoie he writes:

[Le Corbusier] was always endeavouring to open up the house, to create new possibilities for connections between its interior and exterior, and within the interior itself. We want rooms which can be thrown open or enclosed at will, rooms whose outer partitions fall away when we wish. ${ }^{30}$

Giedion uses yet another set of vocabulary to express the quality of Mies van der Rohe's Country House plans:

The analytical spirit of Theo van Doesburg had enabled him to show by means of his transparent architectural drawings that the conception of the house as a self-contained cube had lost its meaning. . . Even more clearly than in the Stijl studies, these country houses of Mies van der Rohe give a realizable form to the floating character of the elements that make up the house. . . Planes protruding from within the house do not halt at the outer walls, as with van Doesburg, but spread out into the landscape like the sails of a windmill . . . ${ }^{31}$

In sum, Giedion attributes three different types of building plans to the three pioneers of modern architecture. According to him, in F. L. Wright a single space is differentiated to accommodate separate functions; in Le Corbusier a dialectics is set up between open spaces and enclosed "rooms"; and in Mies van der Rohe an unconfined space is differentiated by a set of walls.

Like Giedion, Zevi also contrasts the plan which is composed of numerous cubicles to the open plan where spaces are continuously linked:

[The concept of modern space] is based on the open plan. . Internal wall partitions, which no longer serve static bearing functions ... This creates possibility of linking up interior spaces, of joining together the numerous cubicles of the 19th century, of passing from the static plan of the traditional house to the free, open and elastic plan of modern building. . . the open plans offers unlimited possibilities in an isolated building of elastic, internal divisions and subdivisions, either within a rather frozen structure or by means of structure itself. ${ }^{32}$

Additionally, Zevi also compares Le Corbusier, Wright, and Mies to clarify his intuitions regarding different plan types:

[The] Villa Savoie of Le Corbusier and Fallingwater by Wright show clearly the difference in their composition. . . Le Corbusier starts with a reticulated structure, a quadrangle measured regularly by pilasters. Within a rational, geometric formula his space is enclosed by four walls with 
continuous windows. It is only at this point that we begin to deal with the problem of the open plan. The partitions are not static, but formed by thin movable walls. . .

In Mies van der Rohe's [Barcelona Pavilion] . . . the architectural volume is broken up. The continuous space is cut by vertical planes which never form closed, geometrical static areas, but create an uninterrupted flow in the succession of visual angles. Here we have a still freer development of modern themes.

In the case of Wright, aspiration toward spatial continuity has a far more expansive vitality: his architecture is centred around the living reality of interior space. . . [It starts] from a central nucleus ... projecting voids in all directions ... ${ }^{33}$

This attitude to take certain plan types as self-evident can be found even in the more recent configurational studies that emphasize mathematical rigor. For example, Earl and March, in their article "Architectural Applications of Graph Theory" writes:

In floor plans, particularly in domestic dwellings, it is often necessary for individual activity areas to be enclosed by walls. In open plan schemes, however, the walls or partitions define the activity areas, but they do not necessarily define enclosed regions. These open plan schemes are derived from plans with wall enclosing the activity area by the removal of certain walls. ${ }^{34}$

In order to avoid any misunderstanding, these writers, like Zevi and Giedion, use the Villa Malcontenta by Palladio as an example of the plans where the zones are enclosed by walls, and the Farnsworth House by Mies van der Rohe as an example where the zones are not enclosed by walls.

And more than one and a half decades after Earl and March, in his configurational studies Steadman uses a similar intuitive classification as well:

[We] have made a three-way division. The first category is "cellular" space made up of repeated rooms roughly of comparable size, as, for example, hotel bedrooms, individual offices, or school classrooms. The second category is "open plan" space, which is self-explanatory. It is conventional to think of office uses in connection with open plan; but we intend to use the term to apply equally to continuous unobstructed space in warehouse, factories, supermarkets, the sales areas of department stores, and so on.

The third category we have called "halls", by which we mean larger single spaces appropriate for assembly or performances. This category would cover all theatres, cinemas, lecture halls, meeting halls, and other auditoria. It would also apply to law courts, debating chamber, television studios, and churches. There are implications about the visibility of all parts of the hall from every other, or of the stage (pulpit, speaker's rostrum, witness box) from all other points . . ${ }^{35}$ 
However, compared to the other writers cited above, Steadman applies the classification to a broader set of buildings which accommodate very different functions. Steadman's classification draws attention to the fact that an intuitive understanding of building type does not have to be function-specific, and that building plans are often classified based on certain properties of the shape and space irrespective of their functions.

Based on the above survey, it may then be said that architectural theorists have intuitively classified building plans in certain broad categories based on some contrasting properties such as open vs. enclosed space, continuity vs. discontinuity, oneness vs. multiplicity, and interiority vs. exteriority. These plan types can be defined in the following manner without much simplification:

1) The cellular plan type: Any building plan of this type is composed of "rooms" or "cubicles" which are enclosed by walls. It gives a sense of multiplicity within a well-defined bigger whole. Examples of this type may include Palladio's villas.

2) The open plan or free plan type: Any building plan of this type is composed of spaces which are not properly enclosed. Here, the continuous space is articulated by planes which never form closed areas. The plan provides a succession of visual angles rather than uninterrupted conditions of visibility. Here, the relationship between the parts and the whole are intertwined. And very frequently, the whole may lack a sense of limit. Examples of this type may include Mies' Barcelona Pavilion and Brick House. However, in some other examples, the idea of the whole may dominate over the parts. Examples may include Mies' Farnsworth House, Bacardi Office Building in Mexico, and Twentieth Century Gallery in Berlin.

3) The deformed-boundary plan type (or simply deformed type): Any building plan of this type grows out of a single "room" and "projects voids in all direction," to use Zevi's phrase. In the literature, this type of plan is also referred to as the open plan type. However, here they are called deformed plans, because in this type the spaces are created mostly by the articulation of the boundary of the plan. An example of this type may be Wright's Fallingwater. However, some extreme examples of this type may be Steadman's "hall" type plans, which provide uninterrupted conditions of visibility. Examples may include churches, mosques, theatres, cinemas, and lecture halls. In its extreme form, in the deformed plan type the whole dominates over the parts. ${ }^{36}$

Simple plans representing these types for the purposes of this study are difficult to find. Whatever plans are available representing a type generally have widely different number of lines and endpoints. Since the focus of this study is mutual visibility among endpoints, it seems important to somehow control the number of lines and/or endpoints for better comparability. Therefore, three sets of simple artificial plans 
were generated representing three plan types (Figures 10-12). Each cellular plan in figure 10 has 22 interior surfaces as represented by the sides of the lines defining the plan. Four out of the five plans have the same access graph. None of these access graphs has rings. However, these plans are noticeably different in their composition. Each deformed plan in figure $\mathbf{1 1}$ has 16 interior surfaces. There are only three different access graphs for the convex units in the five plans, and none of these access graphs has rings. Again, the compositions of the convex units in these plans are quite different. Each free plan in figure 12 also has 16 interior surfaces. The access graphs of three of these plans have three rings each, while the access graphs of the other two plans has six rings each. Like the other sets, the composition of each plan is quite different from that of the others in the set. In sum, despite their compositional differences the plans in each set have some topological similarities in the way spaces are connected to each other in these plans.

The above characteristics of the plans included in the study become relevant when we consider that one aim of this paper is to find out if the compositional differences among the plans that are topologically similar can be picked by such projective relations as mutual visibility of endpoints in these plans. Now, mutual visibility among the vertices or endpoints of these plans will be studied using different techniques.
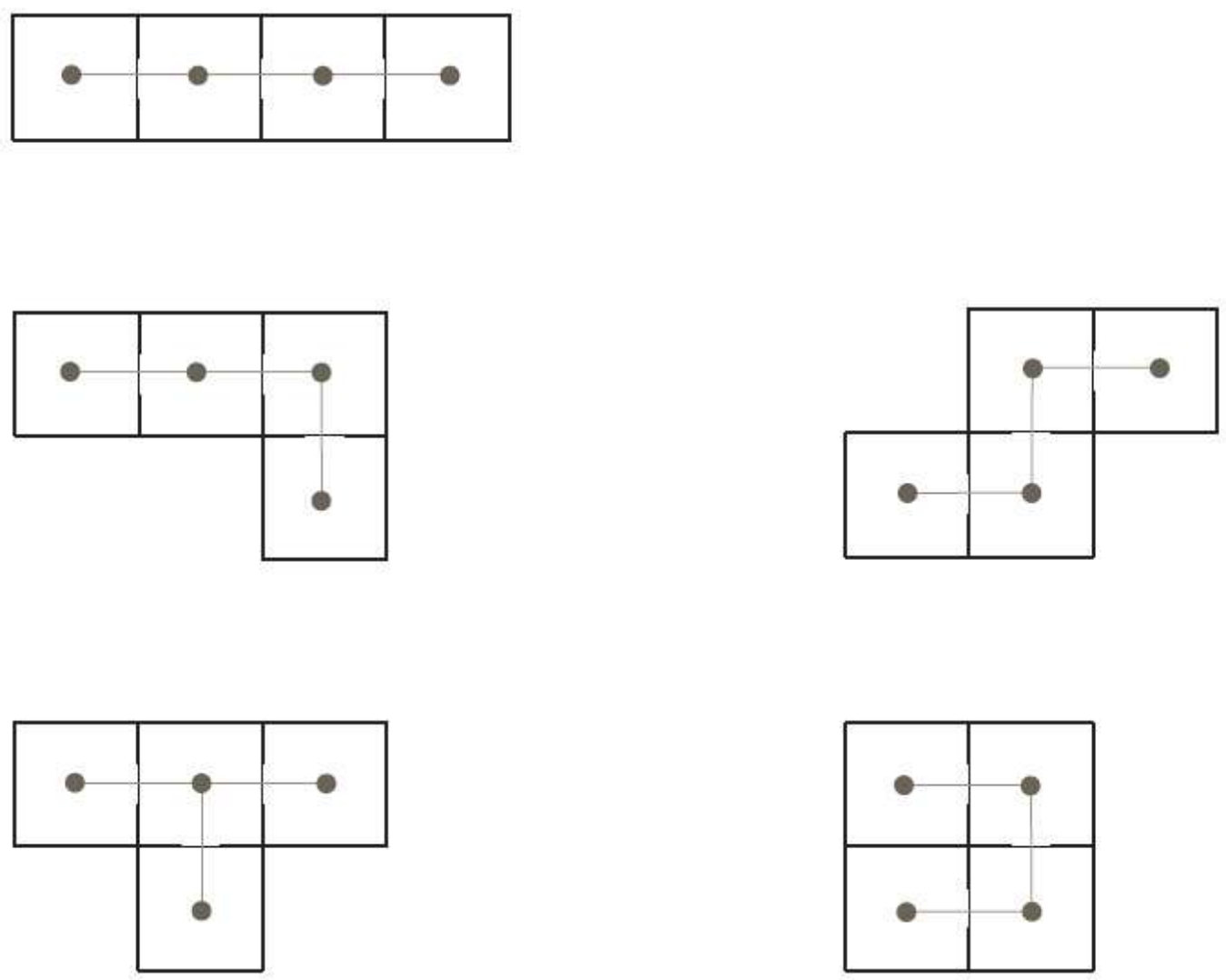

Figure 10: A set of cellular plans, each shown with the access graph on the convex partition. 

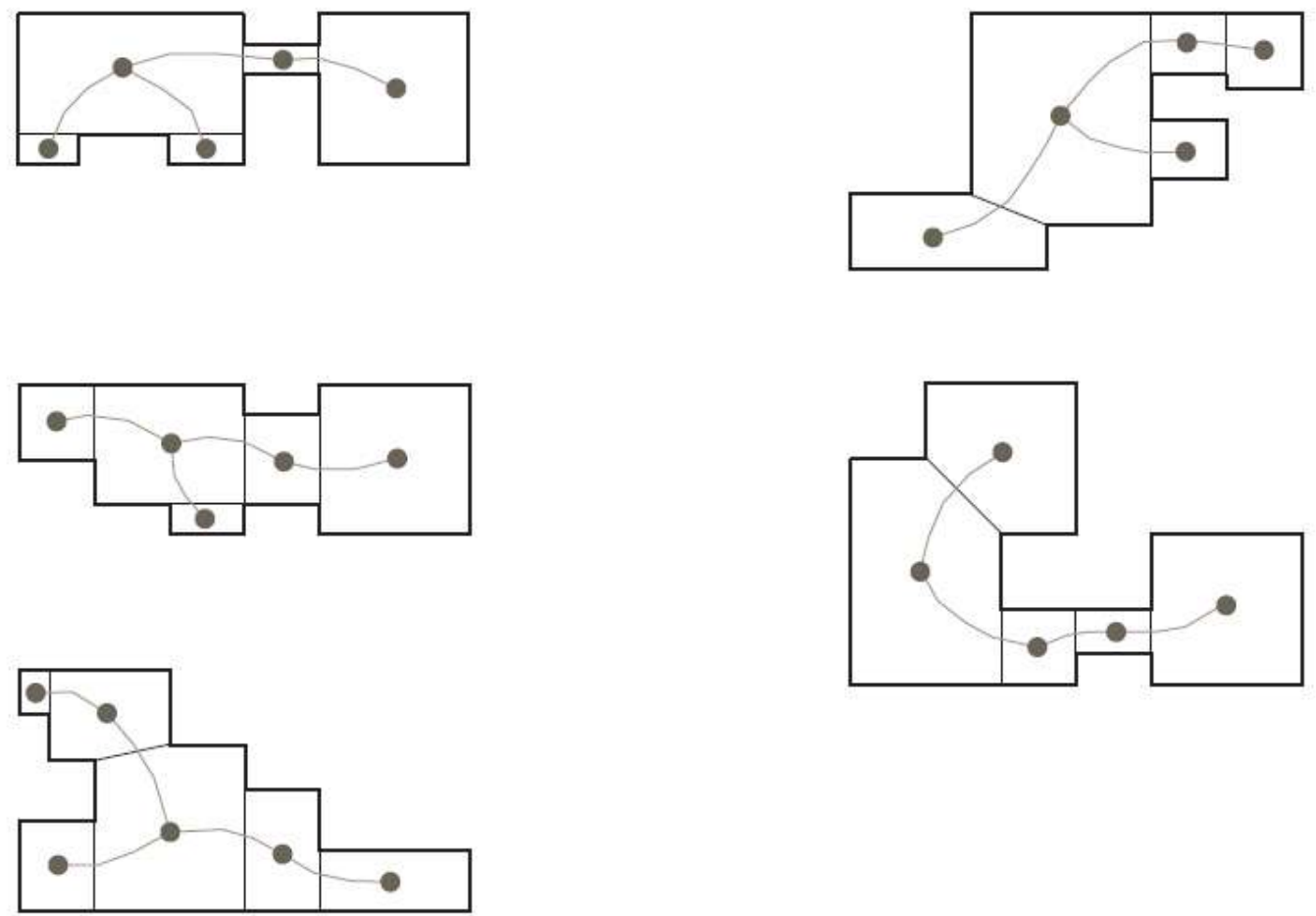

Figure 11: A set of deformed plans, each shown with the access graph on the convex partition.
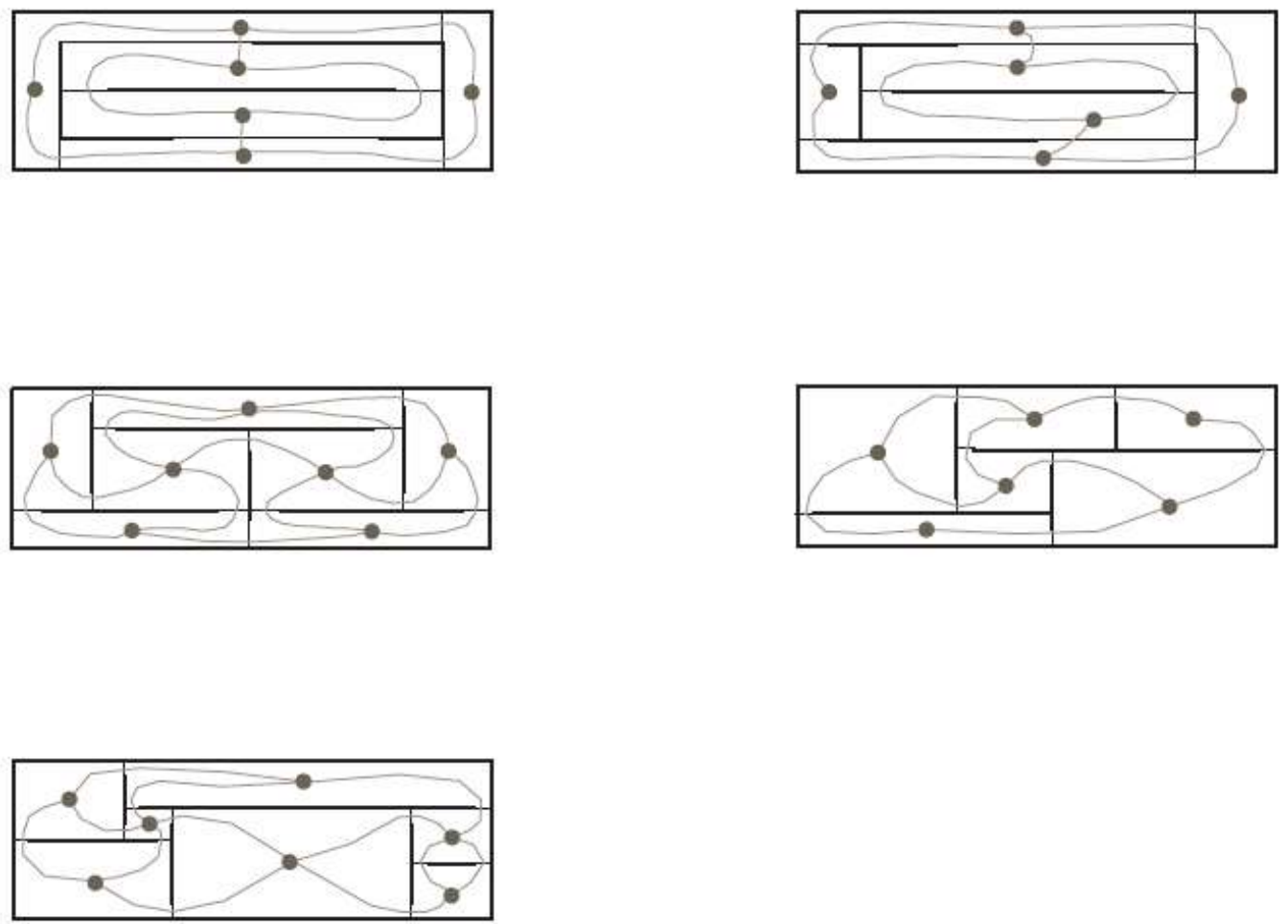

Figure 12: A set of free plans, each shown with the access graph on the convex partition. 


\section{CHARACTERIZING VERTICES IN BUILDING PLANS USING THE CONCEPT OF VISUAL CONNECTIVITY}

In a building plan, lines represent real opaque building walls with surfaces. Depending on its location a line may represent a wall with one or more real surfaces. A vertex is either an endpoint defined by the free end of a line or a corner defined by the intersection of two lines. A vertex does not have any magnitude. Since lines represent real opaque wall, any intersection of two or more lines can have several corners or vertices coincident but hidden from each other. Any two vertices on the same side of a line representing a surface are always adjacent and mutually visible. A vertex can be connected to another vertex in two ways—physically (i.e., by direct access) along the line only, and visually (i.e., by a line of sight) along the diagonals as well as the lines of the plan. Physically, any vertex always has two relations. For example, in the case of a freestanding wall within a plan, each vertex of the line representing the wall has two surfaces incident on it at $360^{\circ}$ apart from each other. Hence, each of them will have two relations to the other vertex. When restricted to their physical relations by direct access only, the graph of the vertices of the shape of a plan is a ring or a set of disjoint rings, where nodes represent the vertices and edges represent the side of the lines connecting the vertices (Figure 13). The physical relations between the vertices of the shape of a plan, then, would seem to bear no value other than the fact that the number of disjoint rings contained within the ring formed by the boundary of the plans may vary from zero to any other positive integer.
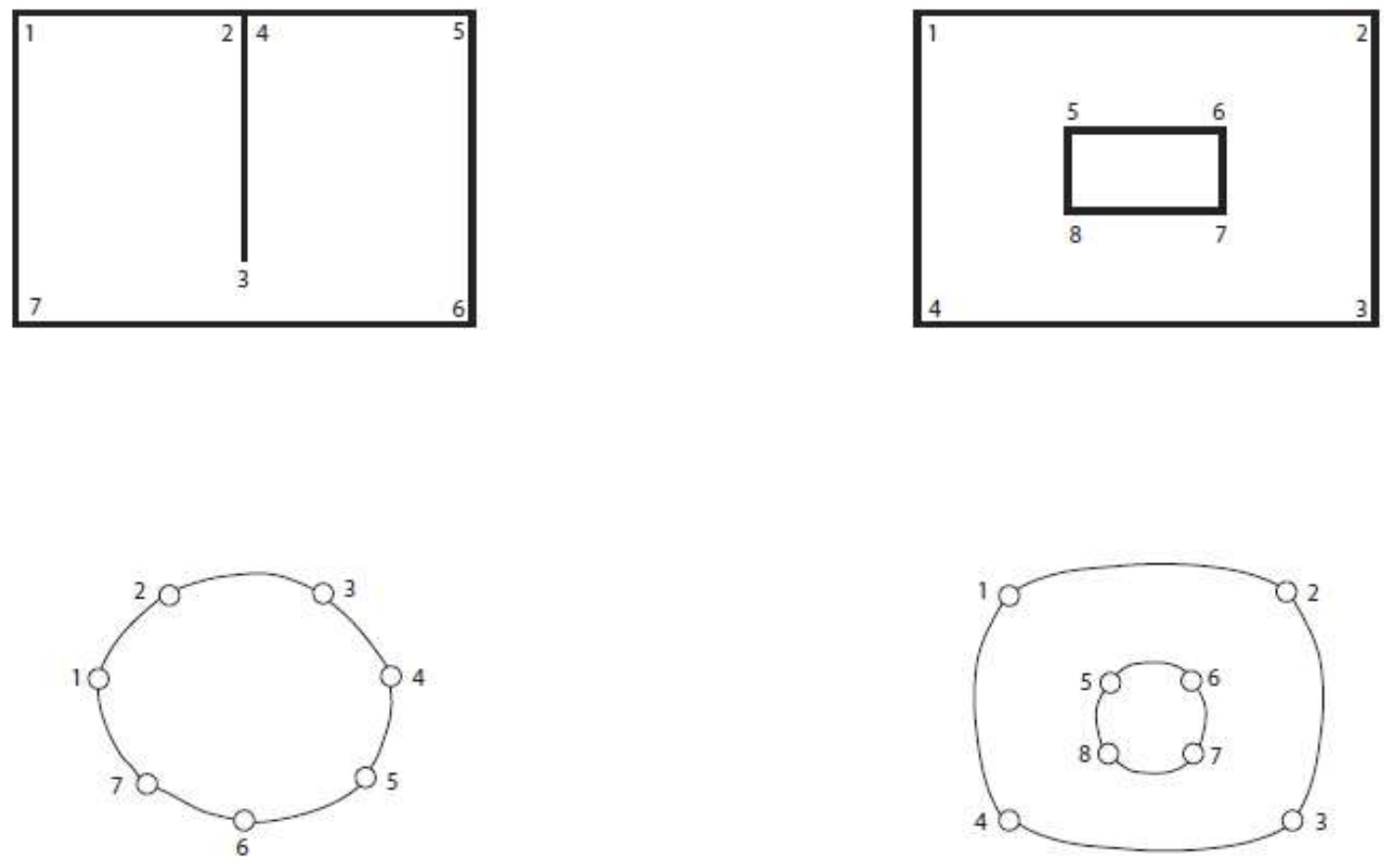

Figure 13: A graph representing the physical relations of vertices in a plan is a ring or a set of disjoint rings. 
In contrast, a vertex may have as many visual relations defined by the lines of sight as the number of diagonals plus the two sides of a line or two lines incident on it. In a plan, visibility among vertices is likely to depend on the disposition of lines in a plan. The visual connectivity of any vertex in a plan is defined as the number of vertices that can be seen from it (Figure 14). In order to compute the visual depth of a vertex, any vertex can be considered one visual-step away from a given vertex if it is directly visible from the given vertex. Likewise, a vertex can be considered two visual-steps away from a given vertex if it is visible only from the vertices that are one visual-step away from the given vertex and are not directly visible from the given vertex. In this manner, we can determine the number of visual-steps that is required to see all the vertices in a plan from any vertex, which can be called the "visual-depth value" of that vertex. Intuitively, a higher visual-depth value of a vertex in a plan would suggest that the vertex is poorly located in the plan in terms of visual relations among its vertices. It is possible that in some plans the distribution pattern of the visual depth values of the vertices is highly differentiated, while in some others this is not the case.

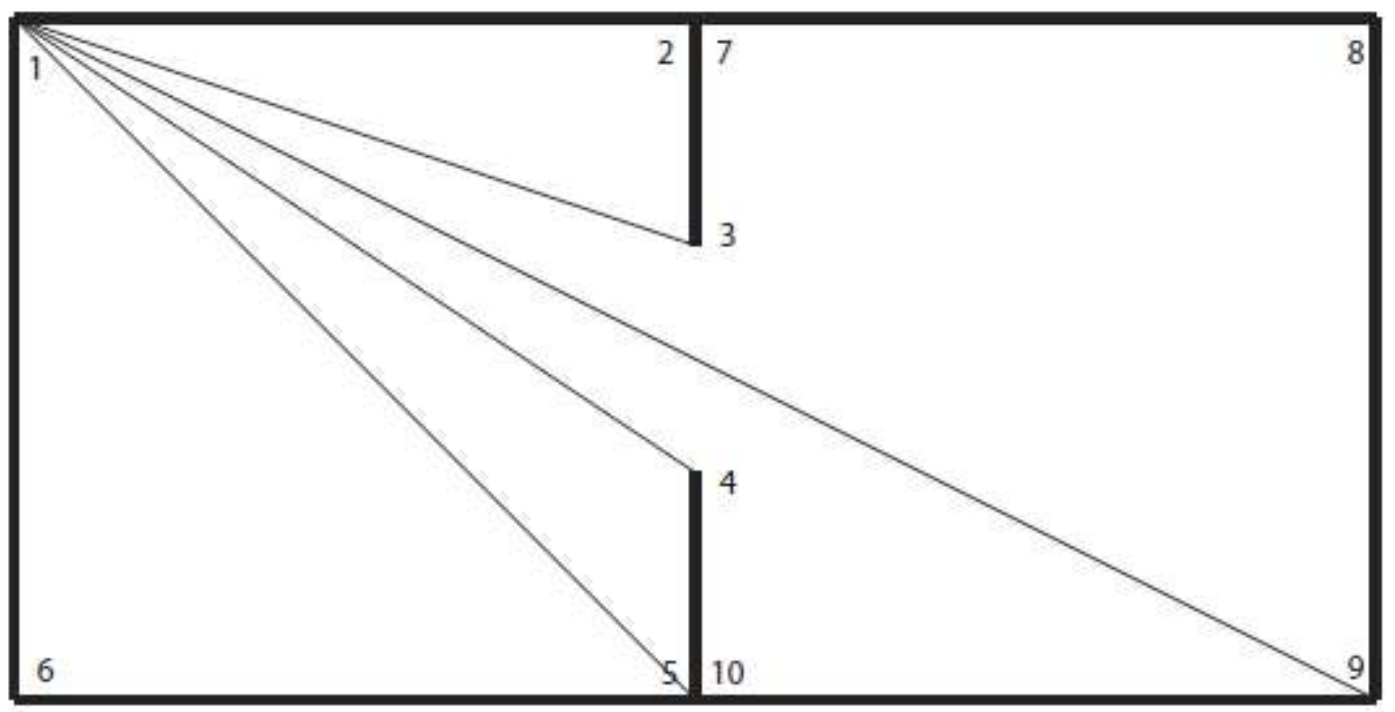

Figure 14: The visual connectivity of any vertex in a plan is the number of vertices that can be seen from it. The set of veritces that can be seen from vertex- 1 is $\{2,3,4,5,6,9\}$. Hence, the visual connectivity value of vertex- 1 is 6 .

The relations of visibility among vertices can also be expressed in a graph, where every vertex in the plan is treated as a node of the graph, and every relation of visibility as a line joining these nodes (Figure 15). Given such a graph, it is possible to draw a justified graph for each vertex in order to determine the visual-depth value of the vertex (Figure 16). Alternatively, one may generate a connectivity matrix containing the list of vertices connected to each vertex in a plan, and then use the matrix to compute 
values of different configurational descriptors of the vertices in the plan using space syntax techniques. ${ }^{37}$ For the purpose of this paper, only two space syntax descriptors are used. Connectivity of an entity is a local property that describes how many entities are directly connected to the given entity, whereas integration is a global property that describes how the entity is connected to all other entities in a system through the entities directly connected to it.

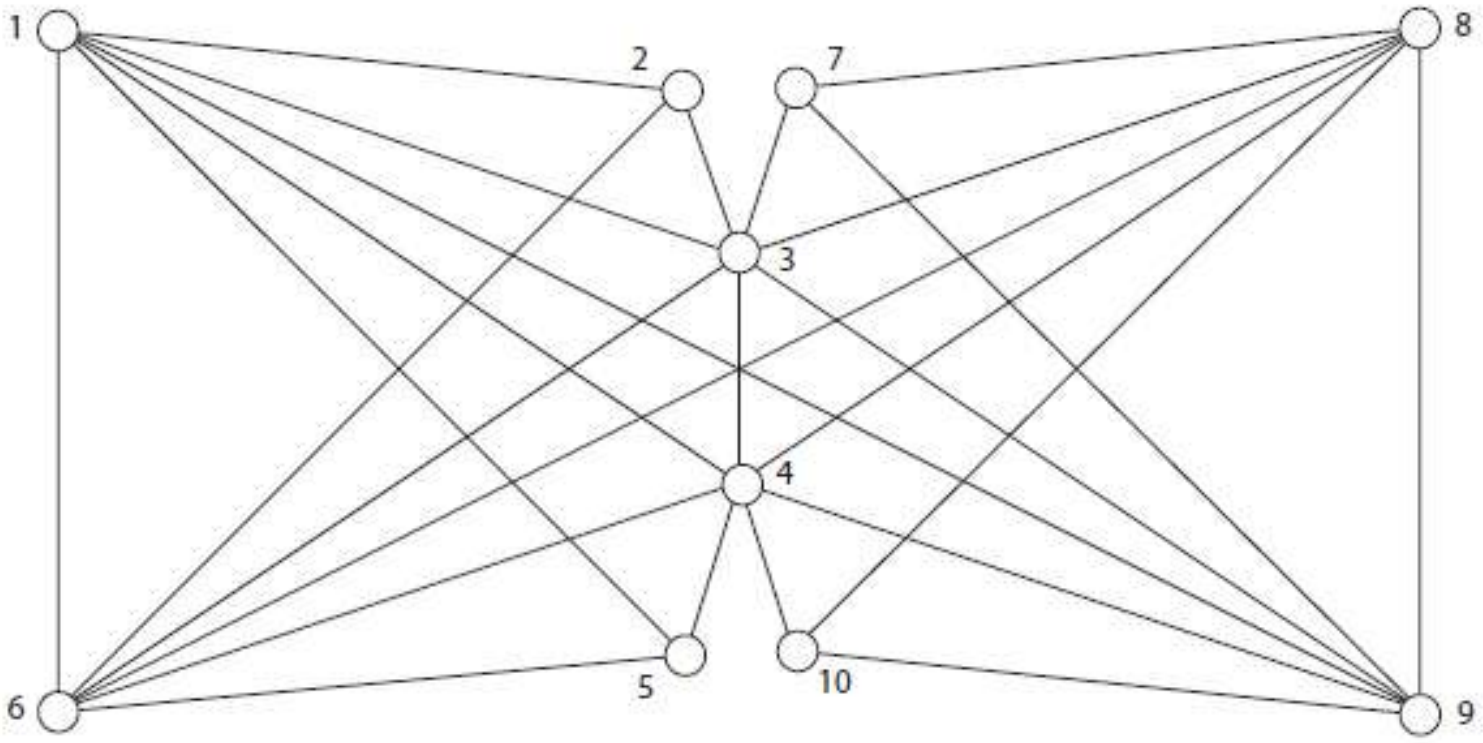

Figure 15: The graph represents the visual relations among vertices in the plan in figure 14.

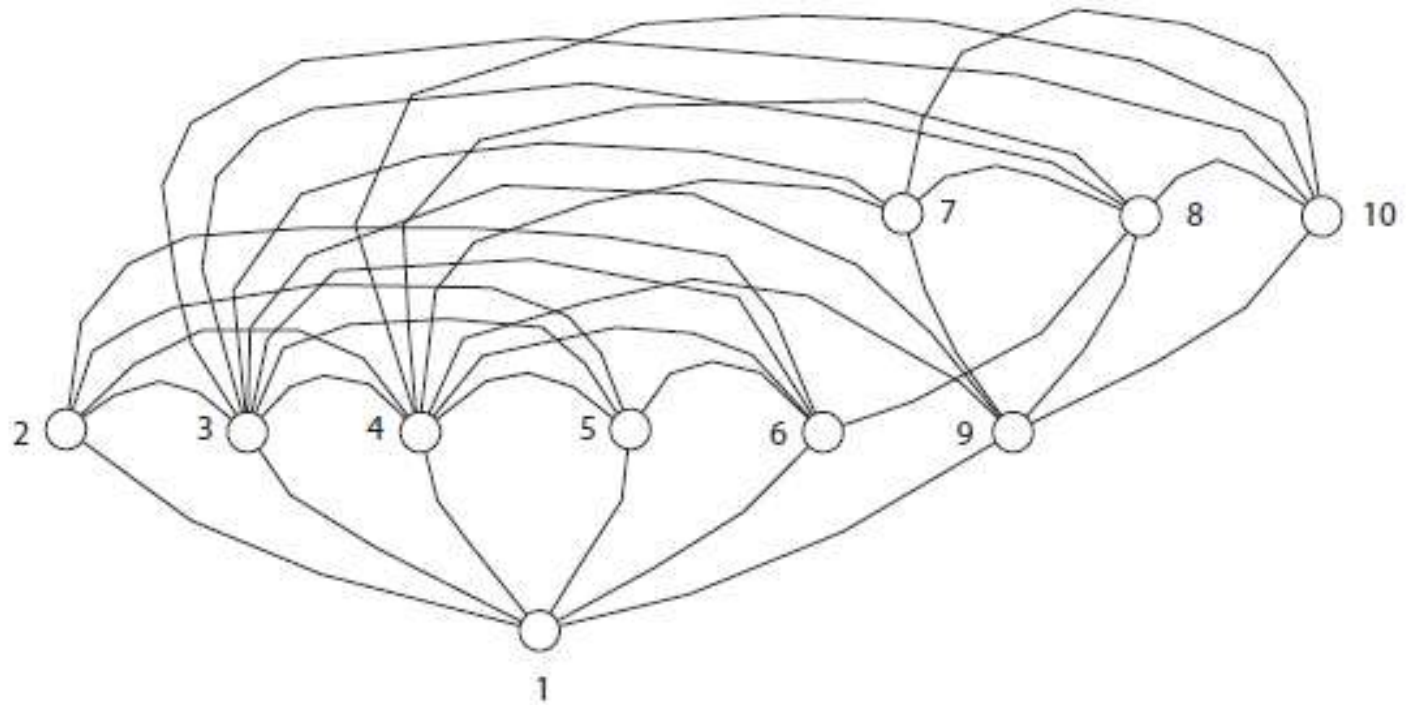

Figure 16: The justified graph for vertex-1.

Note that the number of diagonals and surfaces incident on a vertex may not be equal to the connectivity value of the vertex. In the example of a freestanding wall, every vertex has two sides of the line incident 
on it, but has a connectivity value of one. That is because in this case both sides of the line are connected to the same vertex. In contrast, for a set of several collinear vertices, the number of sides of line-each representing a wall surface and/or the diagonals representing lines of sight from other disjoint vertices incident on any vertex of the set is one, but each vertex of the collinear set contributes to the connectivity value of any other vertex of the set. In other words, if there is a set of $m$ number of collinear vertices in a plan, the connectivity value contributed to each member of the set by the set is equal to $(m-1)$.

The integration value of a vertex computed based on its visual relations with other vertices may allow one to characterize the visibility of a vertex in relation to all other vertices in the plan: The higher the integration value of a vertex the more central its location is in the visual configuration of the vertices in the plan. Figure 17 provides the mean integration and connectivity values, as well as the rank order of vertices according to their integration values in three simple plans representing three different typescellular plan, open/free plan, and deformed plan-each with the same number of vertices. As one would expect, the deformed plan has the highest mean integration values for its vertices, since it provides better visibility among its vertices when compared to the other plans in the figure. However, the fact that the mean integration and connectivity values of the vertices of the cellular plan are more than the free plan is unexpected, but not without reasons. Even though the free plan has freestanding walls allowing spaces to leak onto each other, the disposition of these walls restricts local clustering of the vertices as well as visibility among the vertices. In contrast, the cellular plan has a high number of vertices clustered around local spatial units. In addition, some visibility relations among the vertices in these local units are also allowed through the openings in the cellular plan. As a result, the mean connectivity and integration values of the vertices of the cellular plan are higher than that they are in the free plan.

The mean integration and connectivity values and the pattern of distribution of the integration values of the vertices of the three sets of plans provided in figures 10-12 were also studied. These values are shown in figures 18-20. It can be observed that the free plans allow maximum variations in visibility conditions of their vertices; in contrast, the cellular plans allow minimum variations; and the deformed plans allow in-between conditions. It can also be observed that in plans where visibility relations among vertices are allowed across rooms, the sense of coherence-measured by the variance in the integration values of the vertices in different spatial units-decreases at the local level but increases at the global level. Conversely, where no visibility relation among vertices across rooms is allowed the sense of coherence increases at the local level but decreases at the global level.

Even though vertices represent only a very small segment of all objectively definable points in a plan, as this study already shows, their patterns of mutual visibility can be quite robust to capture interesting properties of the plans. In the next section, mutual visibility of a much larger set of objectively definable points, called the coordination points, will be considered to characterize building plans. 


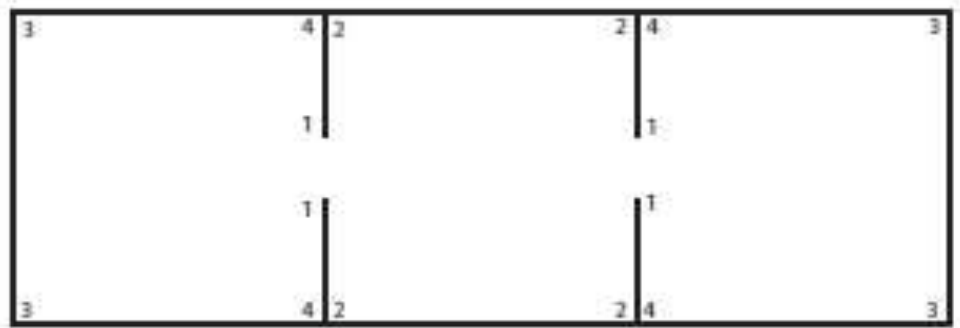

Mnen Intogration: 3409

Muen Comnoctwity: 7.5

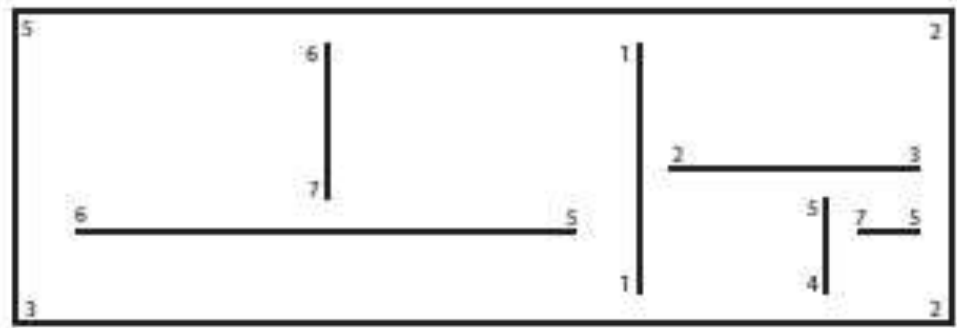

Magn intogration: 2.561

Meen Cocnocthity: 6.625

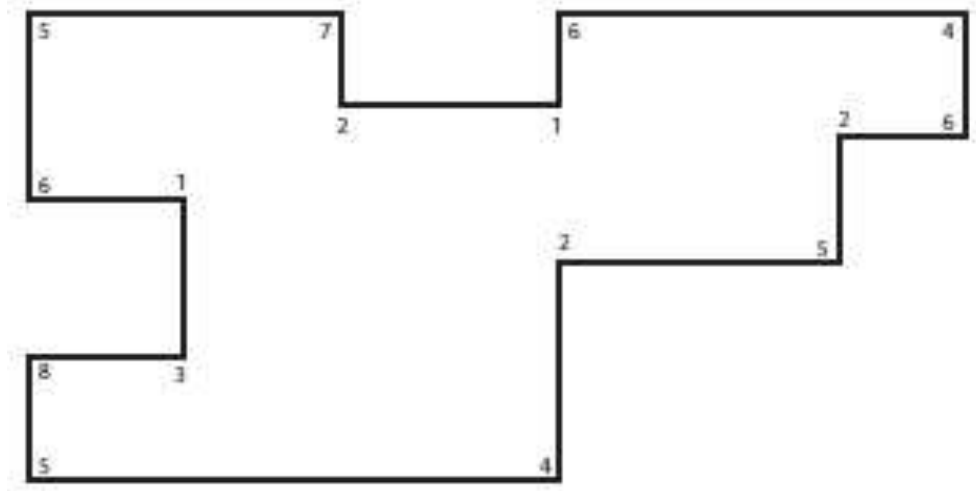

Miran intogration: 455T

Mean Connecthith): 8.5

Figure 17: Rank order of vertices according to their integration values computed on the basis of visual connectivity. Mean integration and mean connectivity values of the vertices in the plans are also provided. 


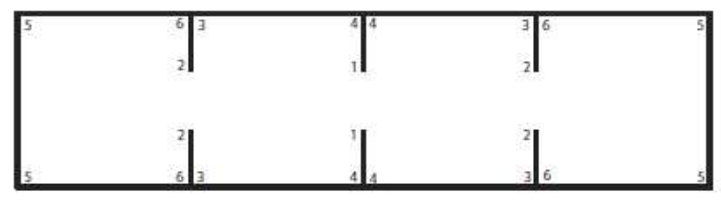

Mean Integration: 3.979 Mean Connectivity: 9.545

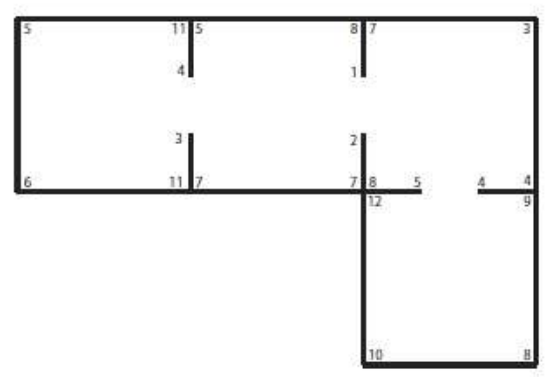

Mean Integration: 3.497

Mean Connectivity: 8.909
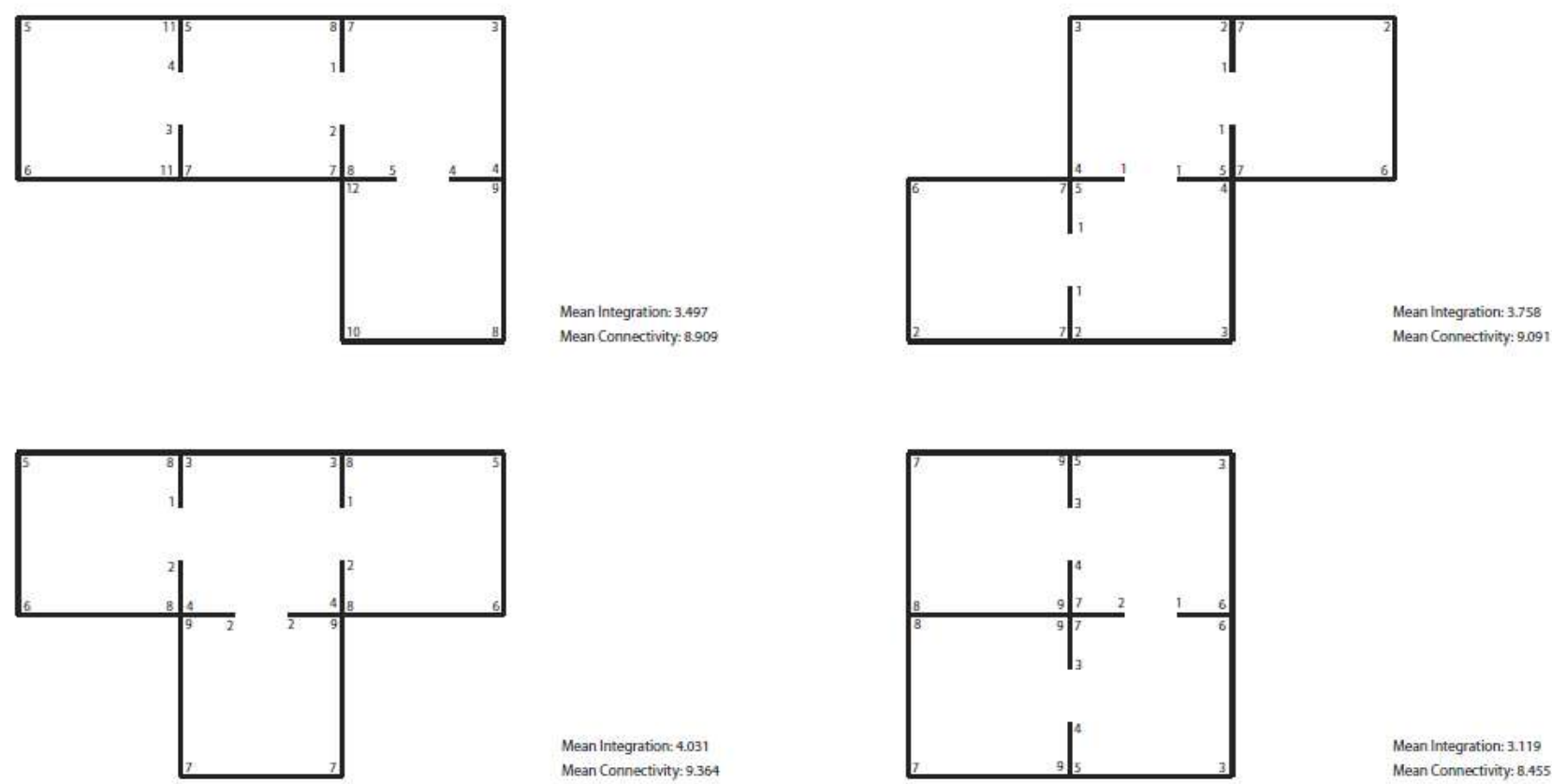

Mean Integration: 4.031 Mean Connectivity: 9.364

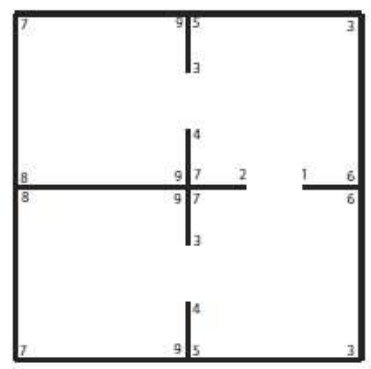

Mean Integration: 3.119 Mean Connectivity: 8.455

Figure 18: Rank order of vertices according to their integration values computed on the basis of visual connectivity.Mean integration and mean connectivity values of the vertices in the plans are also provided. 


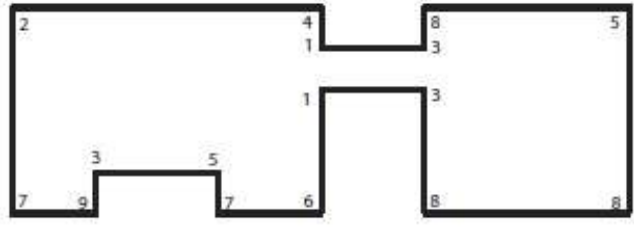

Mean integration: 2828

Mean Connectivity: 6.75

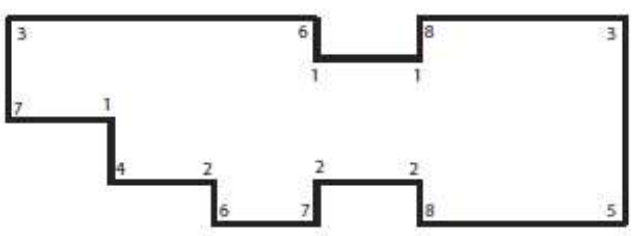

Mean Integration: 4.972

Mean Connectivity: 9

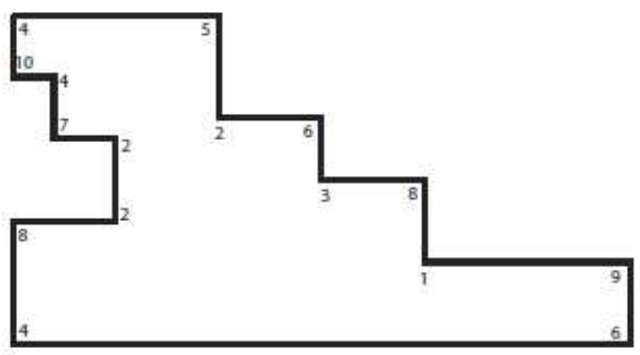

Mean Integration: 3.42

Mean Connectivity: 7.125
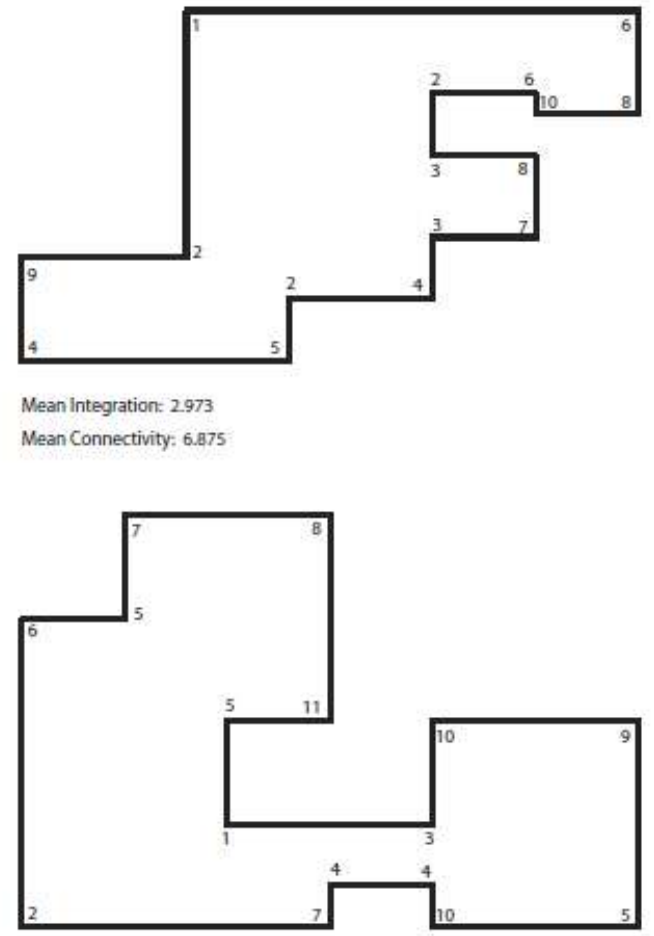

Mean integration: 2.373 Mean Connectivity: 6.25

Figure 19: Rank order of vertices according to their integration values computed on the basis of visual connectivity. Mean integration and mean connectivity values of the vertices in the plans are also provided. 


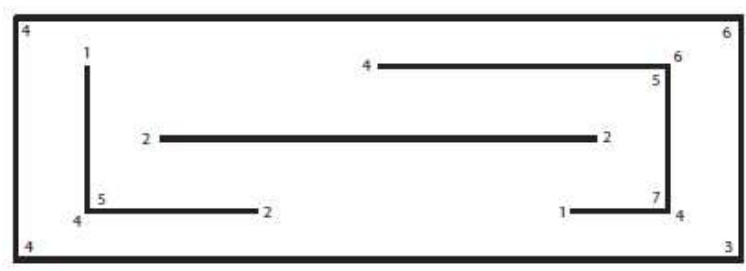

Mean Integration: 3.525

Mean Connectivity: 7.5

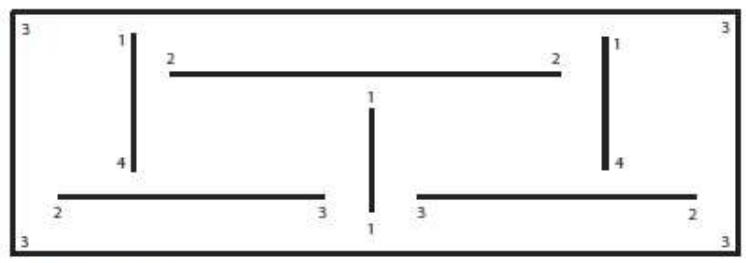

Mean Integration: 3.572

Mean Connectivity: 7.625

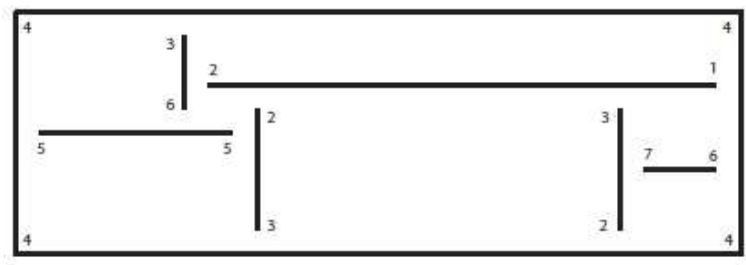

Mean Integration: 3.003

Mean Connectivity: 6.875

Figure 20: Rank order of vertices according to their integration values computed on the basis of visual connectivity.Mean integration and mean connectivity values of the vertices in the plans are also provided.

\section{CHARACTERIZING COORDINATION POINTS IN BUILDING PLANS}

If a point can be connected-either visually by sightlines or physically by direct access along the sides of lines representing walls-to any set of points in a plan, the point may be called a coordination point for the set of points connected to it. There are an infinite number of such coordination points in a plan. Therefore, it may be useful if we can identify a finite set objectively definable points as the coordination points of the plan. For this, any vertex in a plan can be considered as a coordination point for the set of vertices that can be connected to it, either physically or visually. The intersection points of diagonals can also be added to the set of objectively definable coordination points in a plan. Diagonals intersect at vertices, interior points, or both at interior points and vertices of a plan (Figure 21). Any intersection point of diagonals can be treated as a coordination point for the set of vertices connected by the diagonals and/or surfaces incident on the intersection point. While vertices in a plan represent the physically manifest set of coordination points, the intersecting points of diagonals within a plan represent the physically non-manifest set of coordination points. 
In order to study the relationships among the coordination points, both the physically manifest and nonmanifest ones, it is assumed that a coordination point may be connected to other coordination points in two ways. The restricted connectivity of a coordination point-may it be a vertex or an intersection point of diagonals within a plan-is defined as the number of coordination points which can be reached from the given coordination point along a diagonal or along the side of a wall represented as line without crossing any other coordination point. In contrast, the expanded connectivity of a coordination point is defined as the number of coordination points which lie on the surfaces (i.e., the sides of lines representing walls) and the diagonals that are incident on the given coordination point.
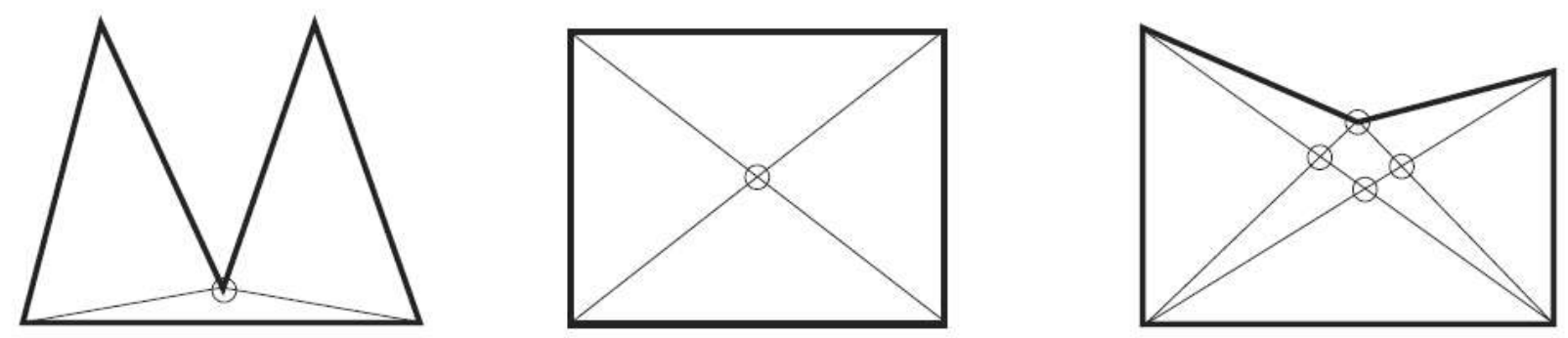

Figure 21: In a plan diagonals may intersect either on vertices, or on points in space, or both on vertices and points in space.

\section{Characterizing coordination points using the concept of restricted connectivity}

In order to describe the configuration of coordination points of a plan based on restricted connectivity, the vertices of a plan as well as the intersecting points of diagonals within the plan are treated as graphnodes. The set of nodes that can be directly connected to a given node along the diagonals and surfaces incident on the given node without crossing any other node are treated as one-step away from the given node. The nodes that can be reached from the one-step away nodes, but not from the origin node, are treated as two steps away from the origin node, and so on. The relationships among these nodes can be expressed in the form of graphs as shown in figures 22-23. A connectivity matrix containing the list of nodes directly connected to each of the nodes of the graph may then be used to compute the integration values of these nodes using space syntax techniques (see above).

Here, note the differences between the configuration of vertices used in the earlier section and the configuration of coordination points composed of the vertices and the intersecting points of diagonals. In the earlier section, a vertex was directly connected to another vertex if they were connected by a diagonal or a surface (i.e., the sides of lines representing walls). The intersecting points of diagonals within the plan were ignored in the configuration. In contrast, here the vertices as well as the intersecting points of diagonals are treated as nodes. Even though two vertices may be connected by a diagonal, the distance 
between them is the number of intervening nodes or the intersecting points that need to be crossed in order to reach a node from the other. If there are several paths to get to a node from another, the path that has the minimum number of intervening nodes will be treated as the distance between the two nodes.
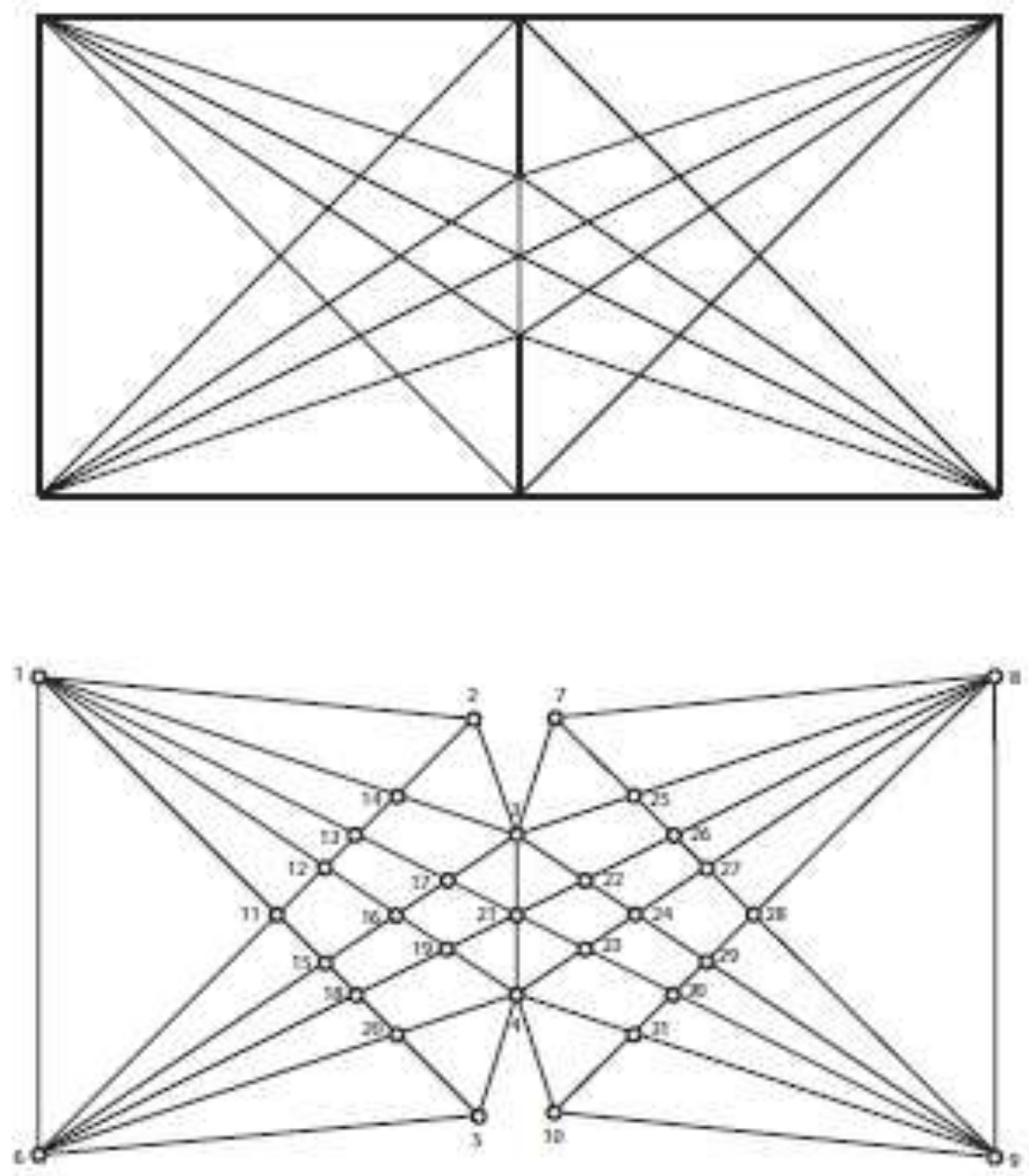

Figure 22 : The graph represents the relations among coordination points as established by the diagonals and surfaces in the plan presented above. 


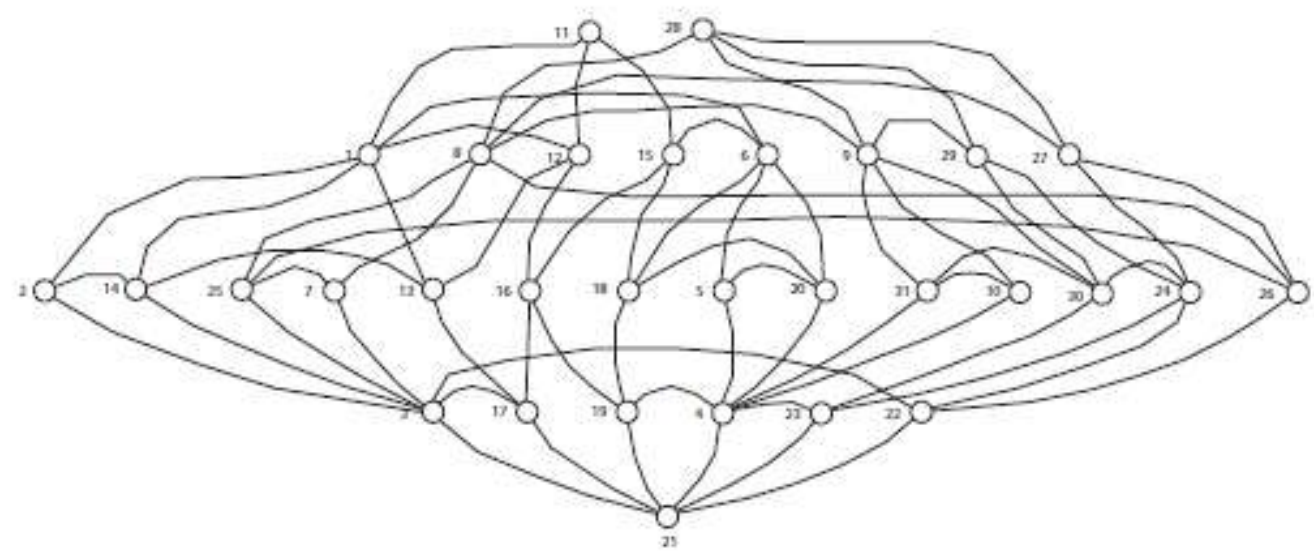

Figure 23: The justified graph for coordination point-21 according to restricted connectivity.

Figures 24-25 show five groups of the most integrated coordination points computed based on restricted connectivity for two sets of simple plans. Though the five groups of the most integrated nodes of these plans were selected for making things a bit easy, they proved sufficient to illustrate some important points of the proposed technique. Note that the plans of each set have the same number of vertices in order to allow a direct comparison, but the disposition of lines representing walls and surfaces in these plans has been manipulated carefully in order to vary some of the relations of incidence among the diagonals. It can be observed that the pattern of distribution of integration values in these plans seems to vary only when the incidence relations of diagonals vary. In other words, metric differences among plans may not change the distribution pattern of integration values if the incidence relations among diagonals remain unchanged. In addition, a comparison between the distribution patterns of the integration values of vertices computed using the concept of visual connectivity with the distribution patterns of the integration values of coordination points computed using the concept of restricted connectivity reveals that for the same set of plans less discrimination is made by the earlier technique. That is because the present technique characterizes a much larger set of nodes that includes the vertices as well as the interior intersecting points of diagonals.

In summary, the analyses suggest that it may be possible to describe the configuration of building plans more precisely if the plans are taken as the configuration of a large set of objectively definable points. They, however, give rise to the following question: Is it then possible to describe the configuration of plans even more precisely if a more elaborate relational pattern is considered among the points in plans? In order to answer the question, the properties of the configuration of the same set of coordination points will be studied next using the concept of expanded visual connectivity. 

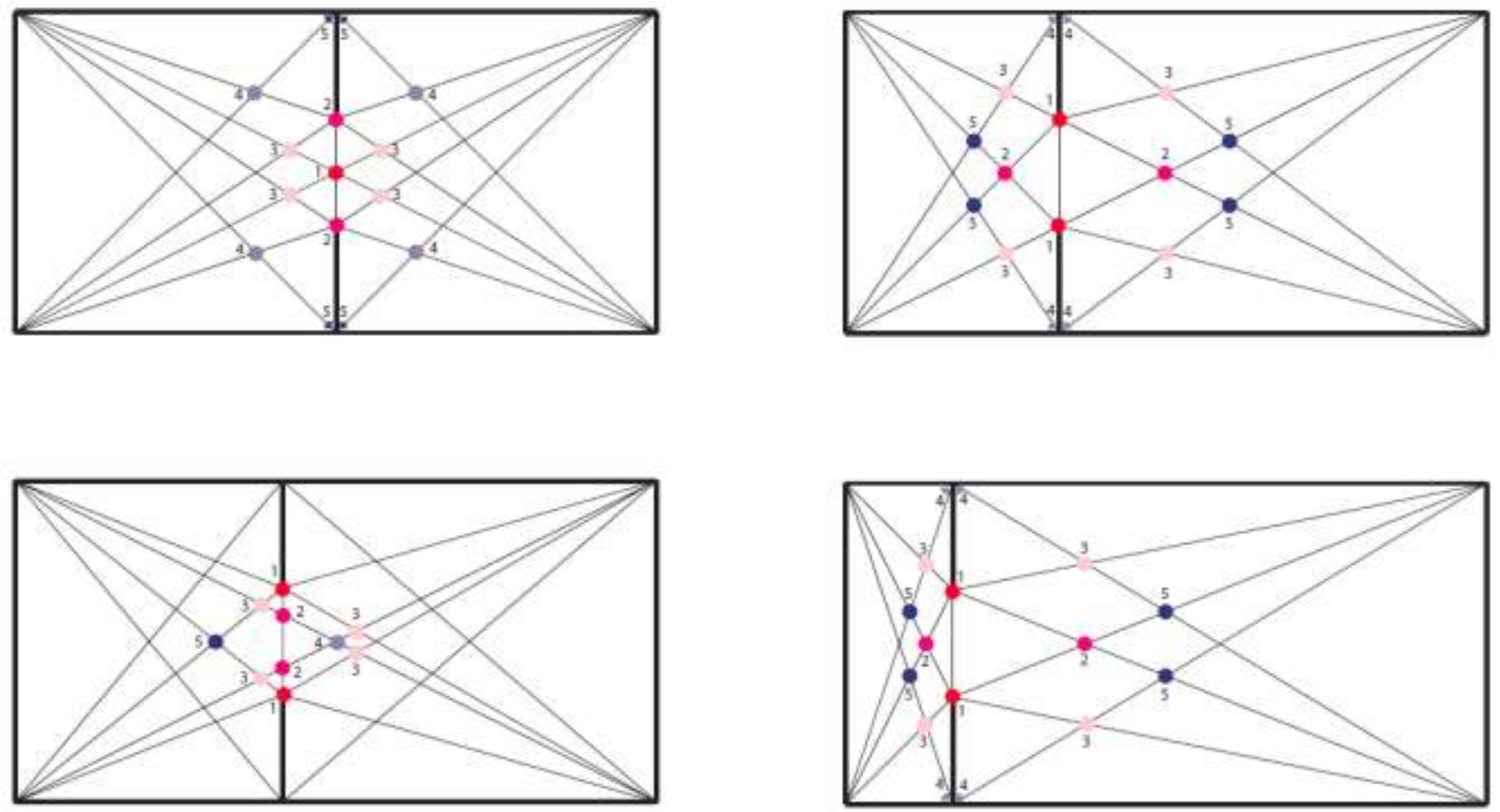

Figure 24: Five groups of most integrated coordination points computed on the basis of restricted connectivity. 

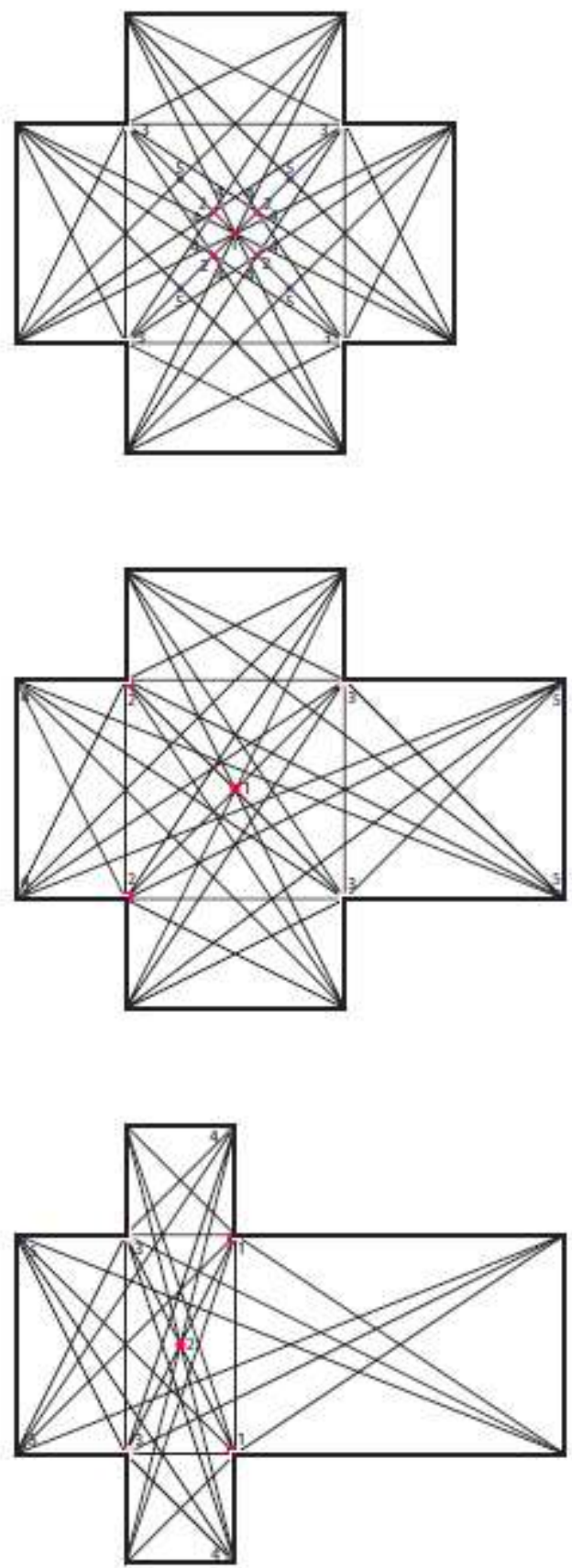

Figure 25: Five groups of most integrated coordination points computed on the basis of restricted connectivity.

\section{Characterizing coordination points using the concept of expanded connectivity}

In order to describe the configuration of coordination points of a plan based on expanded connectivity, the vertices as well as the interior intersecting points of diagonals are treated as graph-nodes. The expanded connectivity of a node, as defined earlier, is the number of nodes that lie on the diagonals and surfaces 
incident on the given node. This set of directly connected nodes is treated to be one-step away from a given node. The nodes which lie on the diagonals and surfaces incident on all the one-step-away nodes, but not on the diagonals and surfaces incident on the origin node, are treated as two steps away from the origin node, and so on (Figures 26-27). Again, using space syntax techniques, the integration value of each node may be computed from the connectivity matrix that contains the list of the directly connected nodes to every node of the graph.
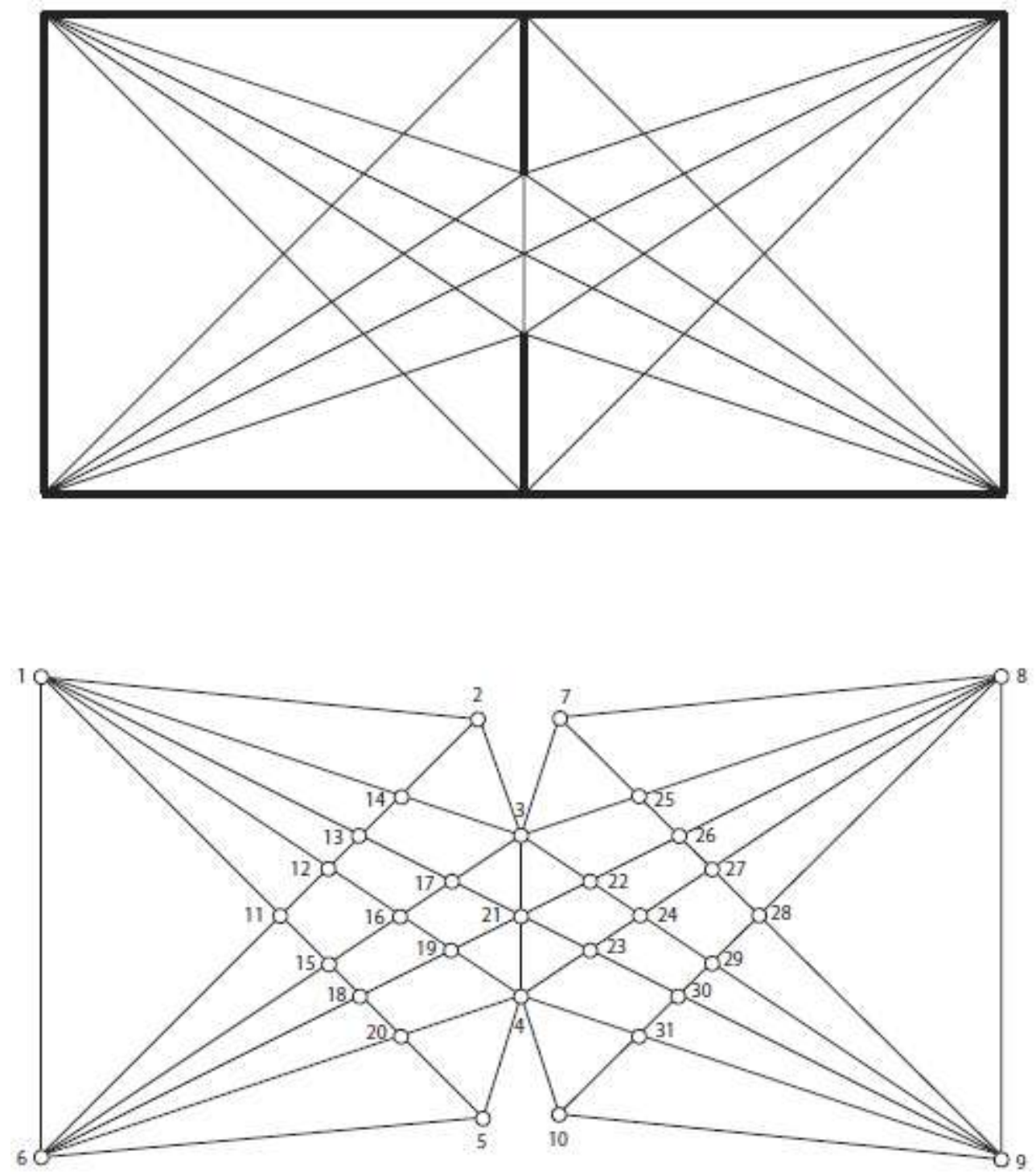

Figure 26: The graph represents the relations among the coordination points as established by the diagonals and surfaces in the plan presented above. 


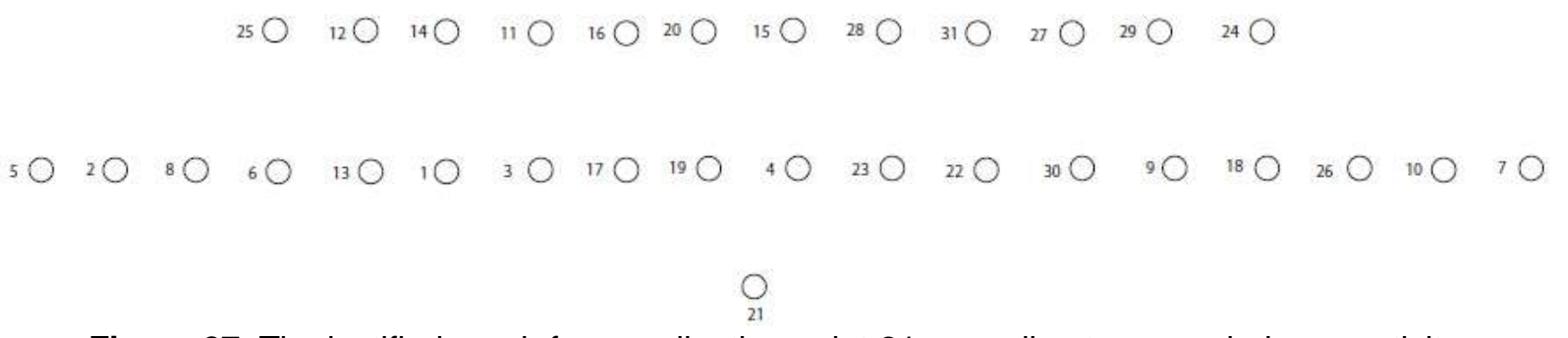

Figure 27: The justified graph for coordination point-21 according to expanded connectivity.

Figures 28-29 represent five groups of the most integrated coordination points computed based on expanded connectivity for two sets of cellular plans only, each set with the same number of vertices. The distribution pattern of integration values computed based on expanded connectivity shows significant differences when compared to that computed based on restricted connectivity in the previous cases. In the previous cases, the pattern of distribution was clustered. The integration value increased or decreased along a directly connected set of points. In the present cases, the pattern of distribution is dispersed. The changes in the integration values are no more limited around a directly connected set of points. More simply, while the distribution pattern of integration values in the previous cases was continuous, in the present cases it is discontinuous.

Note that the present technique differentiates those plans from each other that the earlier technique did not differentiate. That is because in the earlier technique the connectivity value of a point was not related to the number of coordination points along the diagonals and surfaces (i.e., the sides of lines representing walls) that were incident on the point. In contrast, in the present case, the connectivity value of a point changes if the number of coordination points along the diagonals and surfaces that are incident on the point changes. To put it simply, the technique developed based on expanded connectivity would seem to be more shape discriminating than the earlier technique developed based on restricted connectivity.

Note also that the vertices in plans seem to have higher integration values than the interior intersecting points of diagonals when these values are computed using the concept of expanded connectivity (Figures 28-29). That is because in a plan vertices are likely to have more diagonals incident on them; hence, are also likely to have more number of coordination points directly connected to them. In contrast, in restricted connectivity any coordination point- whether it is a vertex or an interior intersecting point of diagonals—has an equal chance of having a high integration value. In other words, the integration values of coordination points computed using the concept of expanded connectivity have a greater ability to discriminate the shapes of plans, but are biased to their vertices. In contrast, the integration values of coordination points computed using the concept of restricted connectivity provide an unbiased description of the configuration of coordination points of a plan, but has a lesser ability to discriminate the shapes of plans. 

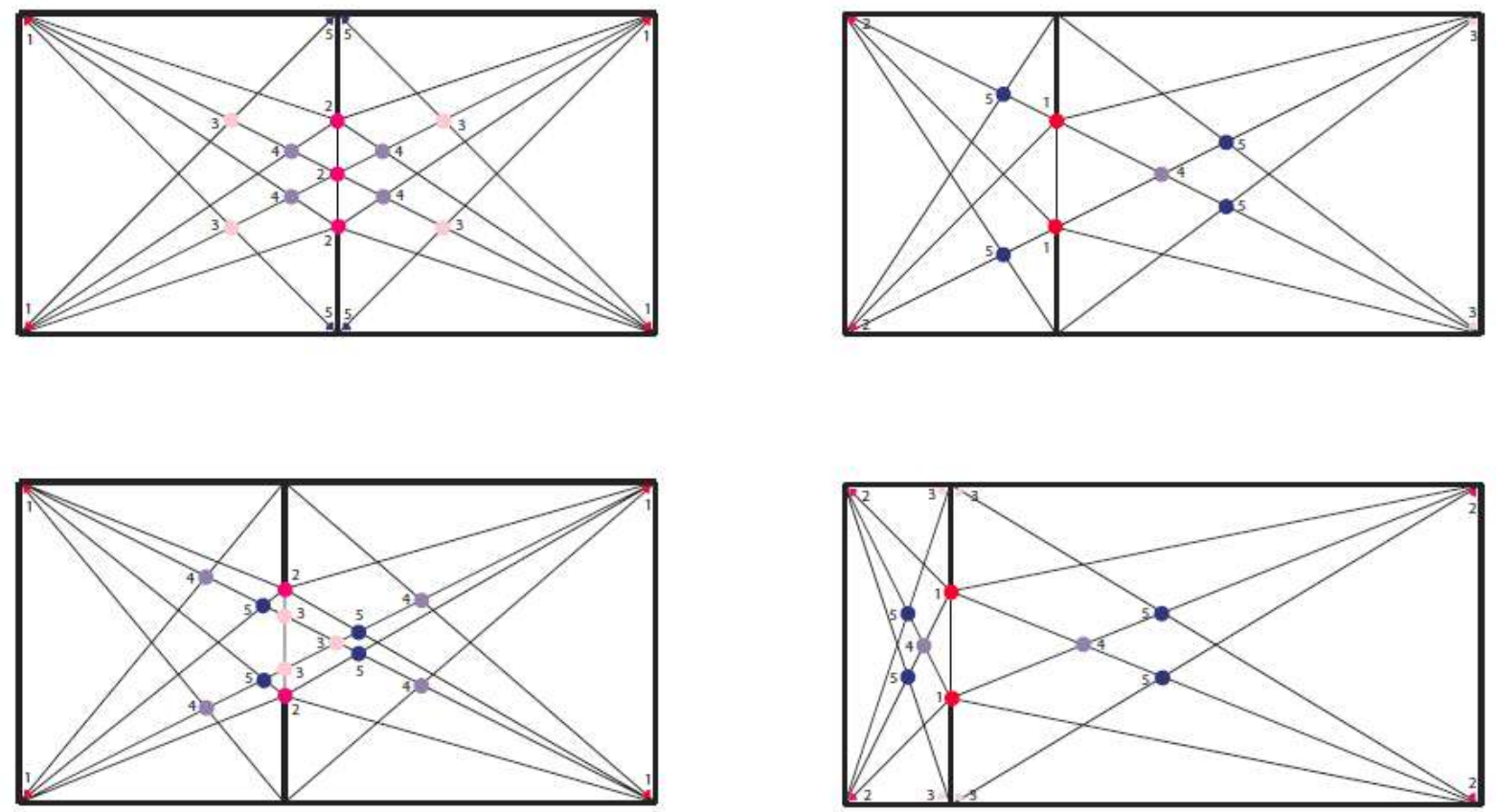

Figure 28: Five groups of most integrated coordination points computed on the basis of expanded connectivity.
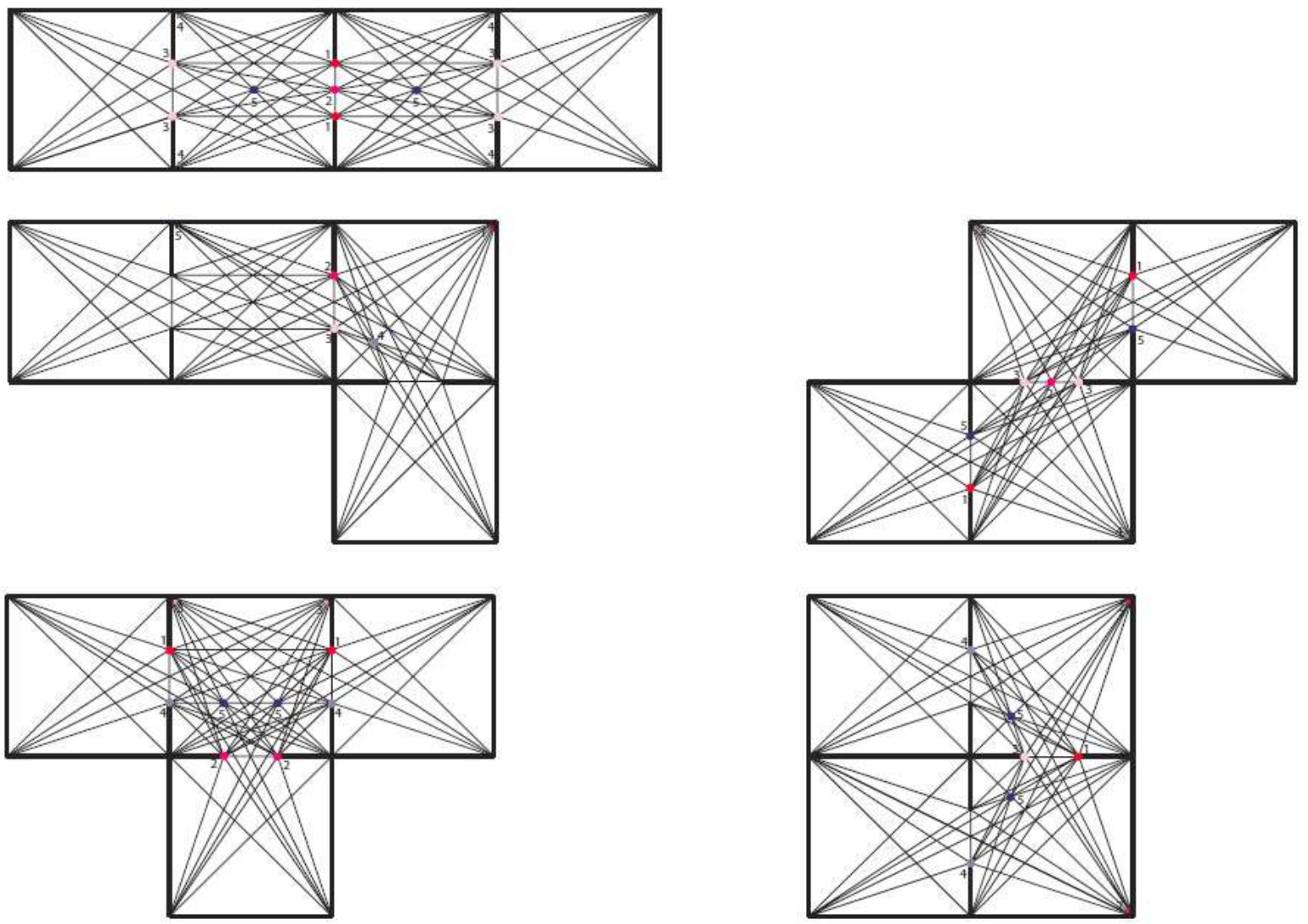

Figure 29: Five groups of most integrated coordination points computed on the basis of expanded connectivity 


\section{CONCLUDING REMARKS}

In this paper, plans were studied based on the patterns of visibility of points defined by the lines and diagonals of the plans. First, visibility patterns of vertices were studied. Following this, visibility patterns of a much larger set of objectively definable points, called the coordination points, were studied. In these studies, visibility relations among points were controlled by imposing different restrictions on a theoretical observer moving inside a plan. It was observed that the graph-theoretic techniques that included larger point sets as well as the techniques that were developed based on an elaborate relational pattern of points were able to make better shape-discrimination. In other words, these techniques show us how, based on visible relations among a set of points, an observer located inside a plan may be able to perceive the differences among plan shapes without having to use any metric relations.

Additionally, these techniques when applied to multiple sets of theoretical plans representing different types revealed intuitively clear typological differences. For example, we expected to see more vertices and corners of the shape in a deformed plan such as the Fallingwater by Frank Lloyd Wright than in a cellular plan such as a villa by Palladio. Descriptions based on mutual visibility of vertices helped clarify this intuition. In contrast, these techniques also revealed interesting counterintuitive typological differences that made perfect sense under careful scrutiny. For example, we expected that a free plan by Mies would have more openness than a cellular plan by Palladio, but we did not expect to find that a free plan would have weaker visibility relations among vertices representing edges of wall surfaces than a cellular plan. Nevertheless, the techniques revealed the unexpected. That is because a free plan suggests continuity more than openness. In a free plan, we see a surface to continue beyond a space but we do not see its edges. Because of this, the space tends to continue beyond its own boundary, better known as the free flowing space. However, the fact that we do not see the edge/s of a surface also gives us a sense of mystery. In other words, free plans heighten our awareness of space by insisting on a sense of continuity as well as a sense of mystery. Indeed, the weaker visibility relations among vertices in free plans helped pick this sense of continuity and mystery of the plans.

One important issue involving plans as configurations of points is the coordination structures of pointsan issue that was not discussed in this paper. A coordination structure of points can be defined as the network of relations among a set of coordination points or positions that provide an economic and unambiguous way to coordinate the vertices of the shape of a plan. ${ }^{38}$ Rashid provides procedures to define a coordination structure for the vertices in a plan based on their visibility relations. ${ }^{39}$ From an analytical viewpoint, a coordination structure that may help coordinate the available information of shape unambiguously into a meaningful whole are important, because they may help us visualize the amount of difficulties involved in the bottom-up understanding of shape. 
Another important issue involving plans as configurations of points not discussed in this paper is the construction of global properties of plan, such as symmetry, from the viewpoint of an observer located inside the plan. In architecture, symmetry is treated as one of the basic global properties of a design composition. However, symmetry of an architectural design becomes clearly manifest only when the design is represented in the form of a plan, because humans are very good at detecting patterns in a planar set which have a finite diameter or which can be viewed at a glance. Therefore, in a real life situation the recognition of any global pattern in architecture may become rather difficult. When located inside a plan, one is never able to appreciate the whole set of synchronic relations that may exist among the elements of the shape of the plan, unless the shape is a very simple convex polygon. Thus, an observer has to construct any global understanding of the plan, such as the symmetry of its shape, from what is given to her diachronically or sequentially as she moves inside the plan. The problem of detecting symmetry of a plan is made more difficult by the fact that the symmetry of a plan is commonly described using the metric relations of its shape. In other words, in order to define symmetry one assumes that one knows the exact measurement of every element of a plan. However, an observer located inside a plan rarely works with the exact knowledge of the metric properties of the shape of a plan. Even if the observer had the knowledge, it is difficult to see how it would help her to understand the symmetry or any other global property of the shape of a plan while she is situated inside the plan.

Using visibility relations of points, Rashid suggests ways in which a situated observer may be able to retrieve the sense of symmetry of a plan. ${ }^{40}$ For this purpose, he distinguishes strong symmetry from weak symmetry-while the first kind uses metric properties, the second kind uses invariant projective and/or topological relations among the elements of a plan. In its weak form, the term symmetry can then be applied to any configuration of points where for every point there is another point with the same relational structure, and where the positions of every such corresponding pair of points may be interchanged without altering the relational structure of the whole set. Since the relational structure of a set of points is stable for a set of plans generated by the isometry, similarity, and affine transformations of the shape of the plans, the construction of symmetry based on configuration of points may remain the same for any member of the set.

Finally, it must be noted here that the techniques presented in this paper provide different unambiguous ways to define centrality in building plans. On the one hand, the visually most integrated set of vertices (i.e., edges of building surfaces) can be treated as centres of building plans in a narrow sense, because vertices do not include locations in the free space that can be occupied by the observer. On the other hand, the visually most integrated set of coordination points can be treated as centres of building plans in a more realistic sense, because they include locations in the free space that can be occupied by the observer. It is easy to explain the importance of a set of visually integrated points in building plans 
borrowing the notion of reference points from spatial cognition. ${ }^{41}$ Since a set of visually integrated points are relatively more visible, they could serve as reference points to define the location of any other adjacent points in a plan. In a sense, it refers to a cognitive system which does not store the relationships between every location in a building. Rather, it stores a set of visually integrated points as reference points and the positions of other points are computed in relation to these points. As a result, these techniques open up the possibility of describing the large-scale environment economically by focusing on a small set of well-defined points.

${ }^{1}$ J. J. Gibson, The ecological approach to visual perception (Houghton Mifflin, Boston, 1979).

2 J. J. Gibson, The senses considered as perceptual systems (Houghton Mifflin, Boston, 1966).

${ }^{3}$ J. J. Gibson, Ibid., p.3.

${ }^{4}$ F. Klein, 'Verglecheinde Betrachtungen "uber neuere geometrischen Forschungen'. Bulletin of the New York Mathematical Society 2 (1893, trans.), pp. 215-249.

${ }^{5}$ J. Piaget, Psychology and Epistemology (Penguin, 1970).

${ }^{6}$ J. Piaget, The mechanisms of perception Trans. by G. N. Seagrim (Basic Books, New York, 1969).

${ }^{7}$ J. Piaget, B. Inhelder, The child's conception of space Trans. by F. J. Langdon, J. L. Lunzer (Routledge \& K. Paul, London, 1967).

${ }^{8}$ G. Franz, J. M. Wiener, 'From Space Syntax to Semantics: A behaviourally and perceptually oriented methodology for the efficient description of the geometry and topology of environments'. Environment and Planning B: Planning and Design, 35 (2008), pp. 574 - 592.

${ }^{9}$ B. Hillier, J. Hanson, The Social Logic of Space (Cambridge, Cambridge University Press, 1984).

${ }^{10}$ J. Peponis, J. Wineman, M. Rashid, S. H. Kim, S. Bafna,, 'On the description of shape and spatial configuration inside buildings: convex partitions and their local properties'. Environment and Planning B: Planning and Design, 24(1997), pp. 761-781.

${ }^{11}$ J. Peponis, J. Wineman, S. Bafna, M. Rashid, S. H. Kim, 'On the Generation of Linear Representations of Spatial Configuration'. Environment and Planning B: Planning and Design, 25 (1998a), pp. 559-576.

12 J. Peponis, J. Wineman, M. Rashid, S. Bafna, S. H. Kim, 'Describing plan configuration according to the co-visibility of surfaces'. Environment and Planning B: Planning and Design, 25 (1998b), pp. 693-708. 
${ }^{13}$ M. L. Benedikt, 'To take hold of space: isovists and isovist fields'. Environment and Planning B: Planning and Design, 6:1 (1975), pp. 47-65.

${ }^{14}$ J. Hanson, 'Deconstructing architects' houses'. Environment and Planning B: Planning and Design, 21(1994), pp. 675-704.

${ }^{15}$ B. Hillier, Space is the Machine (Cambridge University Press, Cambridge, 1996).

${ }^{16}$ B. Hillier, 'Specifically Architectural Knowledge'. The Harvard Architecture Review, 9(1993), pp. 8-27.

${ }^{17}$ A. Turner, M. Doxa, D. O'Sullivan, A. Penn, 'From isovists to visibility graphs: a methodology for the analysis of architectural space'. Environment and Planning B: Planning and Design, 28 (2001), pp. 103121.

${ }^{18}$ B. Hillier, J. Hanson, op. cit.

${ }^{19}$ A. Turner, M. Doxa, D. O'Sullivan, A. Penn, op. cit.

${ }^{20}$ G. Franz, H. A. Mallot, J. M. Wiener, 'Graph-based models of space in architecture and cognitive scienceöa comparative analysis,' in G. E. Lasker, ed., Proceedings of INTERSYMP-2005, 17th International Conference on Systems Research, Informatics and Cybernetics (International Institute for Advanced Studies in Systems Research and Cybernetics, Windsor, Canada, 2005), pp. 30-38.

${ }^{21}$ N. Burgess, 'Spatial cognition and the brain'. Annals of the New York Academy of Sciences, 1124 (2008), pp. 77-97.

22 J. O'Keefe, N. Burgess, 'Geometric determinants of the place fields of hippocampal neurons'. Nature, $381: 6581$ (1996), pp. 425-428.

${ }^{23} \mathrm{~J}$. O'Keefe, 'Place units in the hippocampus of the freely moving rat'. Exp. Neurol., $51: 1$ (1976), pp. 78109.

${ }^{24}$ T. Ono, K. Nakamura, M. Fukuda, R. Tamura, 'Place recognition responses of neurons in monkey hippocampus'. Neurosci. Lett., 121:1-2 (1991), pp. 194-198.

${ }^{25}$ J. O’Keefe, N. Burgess, op. cit.

${ }^{26}$ A. Cressant, R. U. Muller, B. Poucet, 'Failure of centrally placed objects to control the firing fields of hippocampal place cells'. J. Neurosci., 17:7 (1997), pp. 2531-2542.

${ }^{27}$ C. Barry, R. Hayman, N. Burgess, K. J. Jeffery, 'Experience-dependent rescaling of entorhinal grids'. Nat. Neurosci., 10:6 (2007), pp. 682-684. 
${ }^{28}$ C. Barry, C. Lever, R. Hayman, T. Hartley, S. Burton, et al., 'The boundary vector cell model of place cell firing and spatial memory'. Rev. Neurosci., 17 (2006), pp. 71-97.

${ }^{29}$ S. Giedion, Space, Time, and Architecture (Cambridge, MA, Harvard University Press, 1941, reprint 1967), p. 400.

${ }^{30}$ S. Giedion, ibid., p. 525.

${ }^{31}$ S. Giedion, ibid., p. 591.

${ }^{32}$ B. Zevi, Architecture as Space (New York, Horizon Press, 1957, reprint 1974), pp. 141-142.

${ }^{33}$ B. Zevi, ibid., pp. 143-144

${ }^{34}$ C. F. Earl, L. March, "Architectural applications of graph theory," in R. B. Wilson and L. W. Beineke, eds., Applications of Graph Theory (New York, Academic Press, 1979), p. 329.

${ }^{35}$ P. Steadman, 'Built forms and building types: Some speculations'. Environment and Planning B: Planning and Design, 21 (1994), p. s16.

${ }^{36}$ P. Steadman, ibid.

${ }^{37}$ B. Hillier, J. Hanson, op. cit.

${ }^{38} \mathrm{M}$ Rashid, On the configurational studies of building plans from the viewpoint of a situated observer: a partial theory of configuration for plans not involving curves PhD Dissertation (Georgia Institute of Technology, Atlanta, Georgia, 1998).

${ }^{39}$ M. Rashid, ibid.

${ }^{40}$ M. Rashid, op.cit.

${ }^{41}$ E. K. Sadalla, W. F. Burroughs, L. R. Staplin, 'Reference points in spatial cognition'. Journal of Experimental Psychology: Human Learning and Memory, 6:5 (1980), pp. 516-528. 